\title{
BOUNDARY STABILIZATION OF NUMERICAL APPROXIMATIONS OF THE 1-D VARIABLE COEFFICIENTS WAVE EQUATION: A NUMERICAL VISCOSITY APPROACH
}

\author{
AURORA MARICA AND ENRIQUE ZUAZUA
}

\begin{abstract}
In this paper, we consider the boundary stabilization problem associated to the $1-d$ wave equation with both variable density and diffusion coefficients and to its finite difference semi-discretizations. It is well-known that, for the finite difference semi-discretization of the constant coefficients wave equation on uniform meshes 24 or on some non-uniform meshes 20], the discrete decay rate fails to be uniform with respect to the mesh-size parameter. We prove that, under suitable regularity assumptions on the coefficients and after adding an appropriate artificial viscosity to the numerical scheme, the decay rate is uniform as the mesh-size tends to zero. This extends previous results in 24 on the constant coefficient wave equation. The methodology of proof consists in applying the classical multiplier technique at the discrete level, with a multiplier adapted to the variable coefficients.
\end{abstract}

\section{Preliminaries}

Let us consider the following initial boundary value problem associated to the $1-d$ wave equation with variable coefficients and with a damping mechanism acting on the right endpoint of the space interval:

$$
\begin{cases}\rho(x) v_{t t}-\left(\sigma(x) v_{x}\right)_{x}=0, & x \in(0,1), t \in(0, T] \\ v(0, t)=\sigma(1) v_{x}(1, t)+v_{t}(1, t)=0, & t \in[0, T] \\ v(x, 0)=v^{0}(x), v_{t}(x, 0)=v^{1}(x), & x \in(0,1),\end{cases}
$$

where $\left(v^{0}, v^{1}\right) \in H_{l}^{1} \times L^{2}(0,1)$ and $H_{l}^{1}(0,1)=\left\{f \in H^{1}(0,1), f(0)=0\right\}$.

Here we have taken the dissipative boundary condition

$$
\sigma(1) v_{x}(1, t)+v_{t}(1, t)=0,
$$

but similar results can be proved for more general feedback terms as, for instance,

$$
\sigma(1) v_{x}(1, t)+k v_{t}(1, t)=0,
$$

with $k>0$ and $k \neq 1$. In fact, problem (1.1) with the second dissipative condition can be reduced to (1.1) by scaling the time variable.

The energy corresponding to the solution of 1.1 ,

$$
\mathcal{E}_{\rho, \sigma}\left(v(\cdot, t), v_{t}(\cdot, t)\right):=\frac{1}{2}\left(\left\|\sqrt{\rho} v_{t}(\cdot, t)\right\|_{L^{2}}^{2}+\left\|\sqrt{\sigma} v_{x}(\cdot, t)\right\|_{L^{2}}^{2}\right),
$$

obeys the following dissipation law:

$$
\frac{d}{d t} \mathcal{E}_{\rho, \sigma}\left(v(\cdot, t), v_{t}(\cdot, t)\right)=-\left|v_{t}(1, t)\right|^{2} \text { or } \mathcal{E}_{\rho, \sigma}\left(v^{0}, v^{1}\right)-\mathcal{E}_{\rho, \sigma}\left(v(\cdot, t), v_{t}(\cdot, t)\right)=\int_{0}^{t}\left|v_{t}\left(1, t^{\prime}\right)\right|^{2} d t^{\prime} .
$$

When the variable coefficients $\rho$ and $\sigma$ belong to the $B V(0,1)$ class of functions with bounded variation, the following stabilization property holds, ensuring the exponential decay of the energy $\mathcal{E}_{\rho, \sigma}\left(v(\cdot, t), v_{t}(\cdot, t)\right)$

The main idea of this work originated when the first author has visited BCAM in December 2012. Both authors were partially supported by the ERC Advanced Grant FP7-246775 NUMERIWAVES, Grant MTM2011-29306 of MICINN Spain, Project PI2010-04 of the Basque Government, and ESF Research Networking Programme OPTPDE. Additionally, the first author's work was supported by grants MOBIS - Mathematical Optimization and Applications in Biomedical Sciences of the FWF - Austrian Science Fund and PN-II-ID-PCE-2011-3-0075 of CNCS-UEFISCDI Romania. 
of the solutions of (1.1), i.e., the existence of two constants $M, \omega>0$ such that the following estimate holds for any solution $v$ of (1.1) corresponding to the initial data $\left(v^{0}, v^{1}\right) \in H_{l}^{1} \times L^{2}(0,1)$ and any $t>0$ :

$$
\mathcal{E}_{\rho, \sigma}\left(v(\cdot, t), v_{t}(\cdot, t)\right) \leq M \exp (-t \omega) \mathcal{E}_{\rho, \sigma}\left(v^{0}, v^{1}\right) .
$$

There is an extensive literature on the exponential decay of solutions of damped wave equations. In the $1-d$ case under consideration, a careful spectral analysis allows showing that, most often, the eigenfunctions of the associated generator of the semigroup constitute a Riesz basis. This allows characterizing the decay rate in terms of the spectral abscissa. In the case $\rho=\sigma=1$, the solutions of (1.1) vanish in finite time for all $t>2$. But this only occurs in this very exceptional case as pointed out in [5].

For multi-dimensional problems, the analysis of the exponential decay rate cannot be performed by spectral analysis methods and it requires of tools such as microlocal analysis (see the Appendix 2 of [17] by Bardos-Lebeau-Rauch and [1] where the exponential decay is proved by microlocal tools under the so-called Geometric Control Condition), multiplier techniques (19], 27], 28]), and Carleman inequalities [23].

For one-dimensional problems as the one we are considering here, the exponential decay can also be proved by means of the so-called sidewise energy estimates or Liouville transformations ([11], [25], [26]).

In this paper, we are mainly interested in the exponential decay of the finite difference approximations and the multiplier technique seems to be the one that is better adapted to this goal. In [4, in the continuous setting, the variable coefficients case was analyzed and multipliers adapted to the two variable coefficients $\rho$ and $\sigma$ in 1.1 were introduced. Note that the multiplier technique requires some minimal regularity of the coefficients, say, one derivative. This is not just a technical requirement, but rather a necessary condition. Indeed, $B V$ is the minimal regularity assumption on $\rho$ and $\sigma$ to ensure that stabilization holds since, as proved in [3], this property may fail when $\sigma=1$ for some pathological $\rho \in C^{0, \alpha}(0,1)$ for any $\alpha \in(0,1)$.

Consequently, one can say that the exponential decay of solutions of the dissipative wave equation with variable coefficients can be handled in a satisfactory manner using multiplier techniques. But this is far from being the case for numerical approximation schemes and this is the subject we address here. Indeed, the propagation, controllability, and observability properties of the numerical schemes for the wave equation are usually much more delicate to be analyzed due to the existence of fictitious numerical solutions concentrated on the high-frequency modes. These solutions do not affect the convergence of the numerical solutions in the classical sense of the numerical analysis since they are highly oscillatory and weakly converge to zero, but they become important from a stabilization point of view since the standard feedback mechanisms are not capable of dissipating their energy efficiently. Roughly, one can say that boundary feedbacks are inefficient with waves that do not reach the boundary. For the numerical schemes of the constant coefficients wave equations on uniform meshes, the theory is almost complete. Indeed, by now the full panorama of the numerical pathologies and the corresponding remedies to get uniform observability estimates as the mesh size parameter tends to zero are well-understood (see the survey paper [29] or the more recent one [10]).

The paper [24] deals with the finite difference discrete version of the exponential decay estimate 1.3 . under the assumptions that the coefficients $\rho, \sigma$ in the continuous model (1.1) are constant and the grid is uniform. In particular, an uniform exponential decay rate is proved to be recovered uniformly as the mesh size parameter goes to zero by adding an artificial damping in the form of numerical viscosity. This artificial viscosity method is similar to the Tychonoff regularization one introduced by Glowinski-Li-Lions in 13 and proved to be efficient for computing convergent numerical controls for the wave equation. The efficiency of the vanishing viscosity method was proved in some other contexts as well. For example, to recover the uniform controllability properties of a semi-discrete finite difference scheme for the $1-d$ wave equation by means of moment theory tools (fine biorthogonal estimates) in 21]; to obtain uniform stabilization properties of the finite difference semi-discretizations for the perfectly matched layer (PML) approximation of the wave equation in [7. or of some time discretizations of a general class of exponentially stable systems by using the so-called resolvent estimates method in [8] and [9].

The aim of the present paper is to extend the results in [24] to the three-point finite difference semidiscretizations of the wave equation (1.1) with variable and smooth coefficients $\rho, \sigma$ on uniform meshes. We will also see that a numerical scheme on a non-uniform mesh obtained as a diffeomorphic transformation of a uniform one can be written as a numerical scheme for a different variable coefficients wave equation on a uniform mesh. 
Let us also highlight some recent literature on the behaviour of the numerical waves on quasi-uniform meshes or on non-uniform ones obtained by diffeomorphic transformations. In [6, uniform observability properties are obtained for mixed finite element approximations of the constant coefficients wave equation on quasi-uniform meshes. This analysis uses the particular structure of the numerical scheme allowing a full description of the spectrum, even in the non-uniform mesh case. In [2, the spectral distribution of the eigenvalues of sequences of locally Toeplitz matrices arising in numerical approximations of elliptic operators with variable coefficients on non-uniform meshes obtained by diffeomorphic transformations is analyzed. In [20], using pseudo-differential calculus tools, we rigorously define the Fourier symbol and analyze fine geometric properties of the bi-characteristic rays for the finite difference approximations of the variable coefficient wave equation on non-uniform meshes obtained by regular transformations. The spectral distribution in 2 turns out to be the integral on the phase-space domain of the Fourier symbol in 20].

This paper is organized as follows. In Section 2, we recall the stabilization properties of the continuous model. In Subsection 3.1. we state our main result concerning the uniform stabilization of the viscous finite difference approximations of (1.1) and we discuss the analogy between the numerical schemes for the wave equation on non-uniform meshes and those for variable coefficients wave equations on uniform meshes. In Subsection 3.2 we prove the main result in Section 3. Theorem 3.1. and in Subsection 3.3, we test numerically our theoretical result in Subsection 3.1. Finally, in Section 4 we provide some final comments on our results and related open problems. Additionally, for the sake of completeness, we have included Appendix A containing the proof of the main result in Section 2 concerning the continuous model.

\section{Stabilization of the CONTINuOUs MODEL}

The exact statement of the exponential decay property $(1.3)$ is the following one:

Theorem 2.1 (Appendix in [5]). For any strictly positive coefficients $\rho, \sigma \in B V(0,1)$ and any initial data $\left(v^{0}, v^{1}\right) \in H_{l}^{1} \times L^{2}(0,1)$, the solution $v$ of the damped wave equation (1.1) satisfies the estimate (1.3).

By density arguments, it is enough to prove the decay property (1.3) for dense subsets of coefficients and initial data, with constants $M$ and $\omega$ depending on the total variation of the coefficients $\rho$ and $\sigma$. We will consider here the dense subclasses given by strictly positive coefficients $\rho, \sigma \in C^{1}(0,1)$ and initial data $\left(v^{0}, v^{1}\right) \in \mathcal{V}$, where

$$
\mathcal{V}:=\left\{\left(f^{0}, f^{1}\right) \in H_{l}^{1} \times L^{2}(0,1),\left(\partial_{\rho, \sigma}^{2} f^{0}, \partial_{\rho, \sigma}^{2} f^{1}\right) \in H_{l}^{1} \times L^{2}(0,1)\right\}
$$

We denote by $\partial_{\rho, \sigma}^{2} f:=\left(\sigma f_{x}\right)_{x} / \rho$ the weighted Laplace operator involved in the wave equation 1.1$]$. Indeed, set $v_{\epsilon}$ to be the solution of (1.1) corresponding to the strictly positive coefficients $\rho_{\epsilon}, \sigma_{\epsilon} \in C^{1}(0,1)$ and to the initial data $\left(v_{\epsilon}^{0}, v_{\epsilon}^{1}\right) \in \mathcal{V}$ and assume that $\rho_{\epsilon} \longrightarrow \rho, \sigma_{\epsilon} \longrightarrow \sigma$ strongly in $B V(0,1)$ and $\left(v_{\epsilon}^{0}, v_{\epsilon}^{1}\right) \longrightarrow\left(v^{0}, v^{1}\right)$ strongly in $H_{l}^{1} \times L^{2}(0,1)$ as $\epsilon \rightarrow 0$. Taking into account the dissipation law of the energy 1.2 and the uniform positivity of the coefficients $\left(\rho_{\epsilon}, \sigma_{\epsilon}\right)$, we see that $v_{\epsilon}$ is uniformly bounded in $L^{\infty}\left(0, \infty ; H_{l}^{1}(0,1)\right) \cap$ $W^{1, \infty}\left(0, \infty ; L^{2}(0,1)\right)$ as $\epsilon \rightarrow 0$ so that

$$
v_{\epsilon} \rightarrow v \text { weakly star in } L^{\infty}\left(0, \infty ; H_{l}^{1}(0,1)\right) \cap W^{1, \infty}\left(0, \infty ; L^{2}(0,1)\right) .
$$

For any $\theta \in L^{1}\left(0, T ; H_{l}^{1}(0,1)\right) \cap W^{1,1}\left(0, T ; L^{2}(0,1)\right)$, with a finite $T>0, v_{\epsilon}$ satisfies the weak formulation

$$
\left.\int_{0}^{1} \rho_{\epsilon} v_{\epsilon, t} \theta d x\right|_{0} ^{T}-\int_{0}^{T} \int_{0}^{1} \rho_{\epsilon} v_{\epsilon, t} \theta_{\epsilon, t} d x d t+\int_{0}^{T} v_{\epsilon, t}(1, t) \theta(1, t) d t+\int_{0}^{T} \int_{0}^{1} \sigma_{\epsilon} v_{\epsilon, x} \theta_{\epsilon, t} d x d t=0 .
$$

Since $B V(0,1) \subset L^{\infty}(0,1)$ continuously, from the strong convergences in $B V$, we get strong convergence $\rho_{\epsilon} \longrightarrow \rho$ and $\sigma_{\epsilon} \longrightarrow \sigma$ in $L^{\infty}(0,1)$. On the other hand, from the dissipation law $(1.2)$ for $v_{\epsilon}$, we see that $v_{\epsilon, t}(1, t)$ is bounded in $L^{2}(0, T)$ and then, after extracting subsequences, there exists a function $f \in L^{2}(0, T)$ such that $v_{\epsilon, t}(1, \cdot) \rightarrow f$ weakly in $L^{2}(0, T)$. To identify $f$ as $v_{t}(1, \cdot)$, observe that $v_{\epsilon}(1, t)=\int_{0}^{1} v_{\epsilon, x}(x, t) d x$, so that $v_{\epsilon}(1, \cdot) \rightarrow v(1, \cdot)$ weakly in $L^{2}(0, T), v_{\epsilon, t}(1, \cdot) \rightarrow v_{t}(1, \cdot)$ in $H^{-1}(0, T)$ and then $f=v_{t}(1, \cdot) \in L^{2}(0, T)$. We can now rigorously pass to the limit into the weak formulation (2.1) and we obtain that the limit $v$ satisfies the wave equation (1.1) with coefficients $\rho, \sigma$ and initial data $\left(v^{0}, v^{1}\right)$. Taking into account the weak star convergence of $v_{\epsilon}$ to $v$, we obtain $\mathcal{E}_{\rho, \sigma}\left(v(\cdot, t), v_{t}(\cdot, t)\right) \leq \liminf _{\epsilon} \mathcal{E}_{\rho_{\epsilon}, \sigma_{\epsilon}}\left(v_{\epsilon}(\cdot, t), v_{\epsilon, t}(\cdot, t)\right)$, which, combined 
with the passing to the limit in the exponential decay property 1.3 for the regular coefficients $\rho_{\epsilon}, \sigma_{\epsilon}$ and data $\left(v_{\epsilon}^{0}, v_{\epsilon}^{1}\right)$, concludes the exponential decay property $(1.3)$ for all $\rho, \sigma \in B V(0,1)$ and $\left(v^{0}, v^{1}\right) \in H_{l}^{1} \times L^{2}(0,1)$.

Taking into account the above observation, in what follows, we will focus only on the decay property (1.3) for strictly positive coefficients $\rho, \sigma \in C^{1}(0,1)$ and $\left(v^{0}, v^{1}\right) \in \mathcal{V}$. As we will see, the exponential decay property 1.3 is equivalent to the existence of a time $T>0$ and a positive constant $C>0$ such that the following observability inequality holds for any $\left(v^{0}, v^{1}\right) \in \mathcal{V}$ and $v$ the corresponding solution of (1.1):

$$
\mathcal{E}_{\rho, \sigma}\left(v^{0}, v^{1}\right) \leq C \int_{0}^{T}\left|v_{t}(1, t)\right|^{2} d t
$$

Also (2.2) is equivalent to a similar observability property for the corresponding conservative system with initial data $\left(u^{0}, u^{1}\right) \in \mathcal{V}$ :

$$
\begin{cases}\rho(x) u_{t t}-\left(\sigma(x) u_{x}\right)_{x}=0, & x \in(0,1), t \in(0, T] \\ u(0, t)=u_{x}(1, t)=0, & t \in[0, T] \\ u(x, 0)=u^{0}(x), u_{t}(x, 0)=u^{1}(x), & x \in(0,1)\end{cases}
$$

namely,

$$
\mathcal{E}_{\rho, \sigma}\left(u^{0}, u^{1}\right) \leq C^{\prime} \int_{0}^{T}\left|u_{t}(1, t)\right|^{2} d t .
$$

We obtain the following result for which we will give two proofs in Appendix A

Theorem 2.2. $\quad$ a) For all initial data $\left(u^{0}, u^{1}\right) \in \mathcal{V}$ and all strictly positive coefficients $\rho, \sigma \in C^{1}(0,1)$, the observability inequality (2.4) for the solution of (2.3) holds for any time $T$ satisfying

$$
T>\widetilde{T}_{\star}:=2 \ell \text {, with } \ell:=\int_{0}^{1} \sqrt{\frac{\rho(x)}{\sigma(x)}} d x .
$$

b) For all initial data $\left(u^{0}, u^{1}\right) \in \mathcal{V}$ in $(2.3)$, all strictly positive coefficients $\rho, \sigma \in C^{1}(0,1)$ and all $T>0$ the following direct inequality for the solution of (2.3) holds:

$$
\int_{0}^{T}\left|u_{t}(1, t)\right|^{2} d t \leq C^{\prime \prime} \mathcal{E}_{\rho, \sigma}\left(u^{0}, u^{1}\right)
$$

c) Equivalently, the observability inequality (2.2) and the exponential decay estimate (1.3) also hold.

Remark 1. The optimal time $\widetilde{T}_{\star}:=2 \ell$ in (2.5) can be obtained by using the Liouville transformation $\widetilde{x}=L(x):=\int_{0}^{x} \sqrt{\rho / \sigma}\left(x^{\prime}\right) d x^{\prime}$ as in [16] or [25]. In this way, 2.3) is transformed into the wave equation $\widetilde{u}_{t t}-\widetilde{u}_{\widetilde{x} \widetilde{x}}-f_{1}\left(L^{-1}(\widetilde{x})\right) \widetilde{u}_{\widetilde{x}}-f_{0}\left(L^{-1}(\widetilde{x})\right) \widetilde{u}=0$ on the space interval $\widetilde{x} \in(0, \ell)$. Here, the unknown is $\widetilde{u}(\widetilde{x}, t):=$ $u(x, t) / f(x)$ and $f$ can be any strictly positive function such that $f_{1}:=\left((f \sqrt{\rho \sigma})^{\prime}+\sqrt{\rho \sigma} f^{\prime}\right) /(f \rho)$ and $f_{0}:=$ $\left(\sigma f^{\prime}\right)^{\prime} /(f \rho)$ belong to $L^{\infty}(0,1)$. The principal part of this new equation being the d'Alembert operator, the optimal observability time is indeed $\widetilde{T}_{\star}:=2 \ell$.

In this paper, the optimal time $\widetilde{T}_{\star}$ in 2.5 is rigorously obtained by the method of sidewise energy estimates in Appendix A. However, since this method does not seem to be adaptable at the discrete level, we will present a second proof using a multiplier technique, that can be adapted to the discrete case. Note however that the multiplier technique provides the observability property 2.4 in a non-optimal time $T>T_{\star}$,

$$
T_{\star}:=2\|\varphi \sqrt{\rho / \sigma}\|_{L^{\infty}} .
$$

In order to define the function $\varphi$ in (2.7), we first introduce some notations and results concerning the $B V(0,1)$ class. For strictly positive coefficients $\rho, \sigma \in L^{\infty}(0,1)$, we denote by $\rho_{\star}, \rho^{\star}$ and $\sigma_{\star}, \sigma^{\star}$ the four constants with the following properties:

$$
0<a_{\star}:=\inf _{\widetilde{x} \in[0,1]} a(\widetilde{x}) \leq a(x) \leq a^{\star}:=\sup _{\widetilde{x} \in[0,1]} a(\widetilde{x})<\infty \quad \forall x \in[0,1], a \in\{\rho, \sigma\} .
$$


Since $\rho, \sigma \in B V(0,1)$, they admit the Jordan decomposition $a(x):=a(0)+a^{+}(x)-a^{-}(x)$ for all $a \in\{\rho, \sigma\}$ (cf. [12]), where

$$
a^{+}(x):=T V(a, 0, x) \text { and } a^{-}(x):=-a(x)+a(0)+T V(a, 0, x) .
$$

By $T V(a, \alpha, \beta)$, we denote the total variation of the function $a$ on the interval $(\alpha, \beta) \subset(0,1)$. Moreover, $\rho^{+}, \sigma^{+} \geq 0$ and $\rho^{-}, \sigma^{-} \geq 0$ are increasing functions. If $a \in W^{1,1}(0,1) \subset B V(0,1)$, then $T V(a, \alpha, \beta)=$ $\int_{\alpha}^{\beta}\left|a^{\prime}(\widetilde{x})\right| d \widetilde{x}$. Here, ' denotes the derivative of a function depending on one variable.

As indicated above, one of the techniques used here to prove the observability inequality (2.4) with observability time given by (2.7) is the multiplier one (cf. [17]). For $\rho_{\star}, \sigma_{\star}$ and $\rho^{ \pm}, \sigma^{ \pm}$as in (2.8) and (2.9) and inspired by [4], let us define the adapted multiplier $\varphi(x) u_{x}$, where $\varphi$ is given as follows:

$$
\varphi(x):=\exp (\psi(x)), \text { with } \psi(x):=x+\frac{\sigma^{+}(x)}{\sigma_{\star}}+\frac{\rho^{-}(x)}{\rho_{\star}} .
$$

Remark 2. Note that the classical multiplier $x u_{x}$ used to prove observability for the constant coefficient wave equation in [17] is not appropriate for the variable coefficients case. Indeed, by multiplying 2.3) by $x u_{x}$ and integrating in $(x, t) \in(0,1) \times(0, T)$, we obtain the following multiplier identity:

$$
T \mathcal{E}_{\rho, \sigma}\left(u^{0}, u^{1}\right)=\frac{\rho(1)}{2} \int_{0}^{T}\left|u_{t}(1, t)\right|^{2} d t-\left.\mathcal{X}_{x \rho}(t)\right|_{0} ^{T}+\frac{1}{2} \int_{0}^{T} \int_{0}^{1}\left(x \sigma^{\prime}(x)\left|u_{x}(x, t)\right|^{2}-x \rho^{\prime}(x)\left|u_{t}(x, t)\right|^{2}\right) d x d t
$$

where

$$
\mathcal{X}_{\alpha}(t):=\int_{0}^{1} \alpha(x) u_{t}(x, t) u_{x}(x, t) d x .
$$

Using the Cauchy-Schwartz inequality, we get

$$
\left|\mathcal{X}_{x \rho}(t)\right| \leq\|x \sqrt{\rho / \sigma}\|_{L^{\infty}} \mathcal{E}_{\rho, \sigma}\left(u^{0}, u^{1}\right)
$$

so that

$$
\left|\mathcal{X}_{x \rho}(t)\right|_{0}^{T}|\leq 2||x \sqrt{\rho / \sigma}|_{L^{\infty}} \mathcal{E}_{\rho, \sigma}\left(u^{0}, u^{1}\right)
$$

We also easily obtain

$$
\frac{1}{2} \int_{0}^{T} \int_{0}^{1}\left(x \sigma^{\prime}(x)\left|u_{x}(x, t)\right|^{2}-x \rho^{\prime}(x)\left|u_{t}(x, t)\right|^{2}\right) d x d t \leq m_{\rho, \sigma} T \mathcal{E}_{\rho, \sigma}\left(u^{0}, u^{1}\right)
$$

where

- $m_{\rho, \sigma}:=0$, if $\sigma^{\prime} \leq 0$ and $\rho^{\prime} \geq 0$,

- $m_{\rho, \sigma}:=\max \left\{\left\|x \rho^{\prime} / \rho\right\|_{L^{\infty}},\left\|x \sigma^{\prime} / \sigma\right\|_{L^{\infty}}\right\}$, if $\sigma^{\prime} \geq 0$ and $\rho^{\prime} \leq 0$,

- $m_{\rho, \sigma}:=\left\|x \rho^{\prime} / \rho\right\|_{L^{\infty}}$, if $\sigma^{\prime}, \rho^{\prime} \leq 0$,

- $m_{\rho, \sigma}:=\left\|x \sigma^{\prime} / \sigma\right\|_{L^{\infty}}$, if $\sigma^{\prime}, \rho^{\prime} \geq 0$.

Combining identity 2.11) with inequalities 2.13) and 2.14, we get

$$
\left(T\left(1-m_{\rho, \sigma}\right)-2|| x \sqrt{\rho / \sigma} \|_{L^{\infty}}\right) \mathcal{E}_{\rho, \sigma}\left(u^{0}, u^{1}\right) \leq \frac{\rho(1)}{2} \int_{0}^{T}\left|u_{t}(1, t)\right|^{2} d t
$$

so that observability holds under the requirement $m_{\rho, \sigma}<1$ on the coefficients. But this reduces artificially the class of admissible coefficients in Theorem 2.2. 
3. Stabilization of the numerical Approximation scheme: A VAnishing Viscosity Method

3.1. Main result. Consider $N \in \mathbb{N}, h:=1 /(N+1)>0$ to be the mesh size parameter and $\mathcal{G}^{h}:=\left\{x_{j}=\right.$ $j h, 0 \leq j \leq N+1\}$ an uniform grid of size $h$ of the interval [0,1]. For $\sigma$ as in (1.1), set $\sigma_{j+1 / 2}:=\sigma\left(x_{j+1 / 2}\right)$, with $0 \leq j \leq N$, and $\rho_{j}:=\rho\left(x_{j}\right)$, with $0 \leq j \leq N+1$. We denote by $\mathbf{f}^{h}$ the column vector of components $f_{j}, 0 \leq j \leq N+1$. Also define the forward, backward and centered first-order finite differences $\partial_{+}^{h}, \partial_{-}^{h}$ and $\partial^{h}$ as $\partial^{h, \pm} f_{j}:= \pm\left(f_{j \pm 1}-f_{j}\right) / h$ and $\partial^{h} f_{j}:=\left(f_{j+1}-f_{j-1}\right) /(2 h)$. Let $\partial_{\sigma}^{h, 2}$ be the three point finite difference approximation of the weighted second-order derivative $\partial_{\sigma}^{2}:=\partial_{x}\left(\sigma \partial_{x}\right)$ defined as follows:

$$
\partial_{\sigma}^{h, 2} f_{j}:=\frac{\sigma_{j+1 / 2} \partial_{+}^{h} f_{j}-\sigma_{j-1 / 2} \partial_{-}^{h} f_{j}}{h} .
$$

We also set $\partial^{h, 2}:=\partial_{1}^{h, 2}$ to be the classical centered three point finite difference operator approximating the Laplacian $\partial_{x x}$.

Let us now consider the following viscous finite difference semi-discretization of the damped wave equation (1.1) on the uniform grid $\mathcal{G}^{h}$ :

$$
\begin{cases}\rho_{j} v_{j}^{\prime \prime}(t)-\partial_{\sigma}^{h, 2} v_{j}(t)=h^{2} \partial^{h, 2} v_{j}^{\prime}(t), & 1 \leq j \leq N, t \in(0, T] \\ v_{0}(t)=\sigma_{N+1 / 2} \partial_{+}^{h} v_{N}(t)+v_{N+1}^{\prime}(t)=0, & t \in[0, T] \\ \mathbf{v}^{h}(0)=\mathbf{v}^{h, 0},\left(\mathbf{v}^{h}\right)^{\prime}(0)=\mathbf{v}^{h, 1} . & \end{cases}
$$

In this section, ' denotes the time derivative. The energy associated to the damped wave equation (3.2),

$$
\widetilde{\mathcal{E}}_{\rho, \sigma}^{h}\left(\mathbf{v}^{h}(t), \mathbf{v}_{t}^{h}(t)\right):=\frac{h}{2} \sum_{j=1}^{N} \rho_{j}\left|v_{j}^{\prime}(t)\right|^{2}+\frac{h}{2} \sum_{j=0}^{N} \sigma_{j+1 / 2}\left|\partial_{+}^{h} v_{j}(t)\right|^{2}+\frac{h^{2}}{2 \sigma_{N+1 / 2}}\left|v_{N+1}^{\prime}(t)\right|^{2},
$$

is decreasing in time and satisfies the following dissipation law:

$$
\frac{d}{d t} \widetilde{\mathcal{E}}_{\rho, \sigma}^{h}\left(\mathbf{v}^{h}(t), \mathbf{v}_{t}^{h}(t)\right)=-\left|v_{N+1}^{\prime}(t)\right|^{2}-h^{3} \sum_{j=0}^{N}\left|\partial_{+}^{h} v_{j}^{\prime}(t)\right|^{2}
$$

or

$$
\widetilde{\mathcal{E}}_{\rho, \sigma}^{h}\left(\mathbf{v}^{h}(0), \mathbf{v}_{t}^{h}(0)\right)-\widetilde{\mathcal{E}}_{\rho, \sigma}^{h}\left(\mathbf{v}^{h}(t), \mathbf{v}_{t}^{h}(t)\right)=\int_{0}^{t}\left|v_{N+1}^{\prime}\left(t^{\prime}\right)\right|^{2} d t^{\prime}+h^{3} \sum_{j=0}^{N} \int_{0}^{t}\left|\partial_{+}^{h} v_{j}^{\prime}\left(t^{\prime}\right)\right|^{2} d t^{\prime} .
$$

As in the continuous case, we are interested in the exponential decay property of the discrete wave equation 3.2 , i.e. in the existence of two positive constants $\widetilde{M}, \widetilde{\omega}>0$ independent of $h$ such that the following energy estimate holds for all $t>0$ and for all initial data $\left(\mathbf{v}^{h, 0}, \mathbf{v}^{h, 1}\right)$ in $(3.2)$ :

$$
\widetilde{\mathcal{E}}_{\rho, \sigma}^{h}\left(\mathbf{v}^{h}(t), \mathbf{v}_{t}^{h}(t)\right) \leq \widetilde{M} \exp (-t \widetilde{\omega}) \widetilde{\mathcal{E}}_{\rho, \sigma}^{h}\left(\mathbf{v}^{h, 0}, \mathbf{v}^{h, 1}\right)
$$

Also, as for the continuous model, the decay property 3.4 is equivalent to the existence of a finite time $T>0$ and of a constant $C>0$ such that the following observability estimate holds for any solution $\mathbf{v}^{h}(t)$ of the damped system 3.2 , uniformly as $h \rightarrow 0$ :

$$
\widetilde{\mathcal{E}}_{\rho, \sigma}^{h}\left(\mathbf{v}^{h}(0), \mathbf{v}_{t}^{h}(0)\right) \leq C\left(\int_{0}^{T}\left|v_{N+1}^{\prime}(t)\right|^{2} d t+h^{3} \sum_{j=0}^{N} \int_{0}^{T}\left|\partial_{+}^{h} v_{j}^{\prime}(t)\right|^{2} d t\right) .
$$

Consider the following finite difference approximation of $(2.3)$ :

$$
\begin{cases}\rho_{j} u_{j}^{\prime \prime}(t)-\partial_{\sigma}^{h, 2} u_{j}(t)=0, & 1 \leq j \leq N, t \in(0, T] \\ u_{0}(t)=\partial_{+}^{h} u_{N}(t)=0, & t \in[0, T] \\ \mathbf{u}^{h}(0)=\mathbf{u}^{h, 0},\left(\mathbf{u}^{h}\right)^{\prime}(0)=\mathbf{u}^{h, 1}, & \end{cases}
$$

for which the total energy of the solutions given below is time conservative

$$
\mathcal{E}_{\rho, \sigma}^{h}\left(\mathbf{u}^{h}(t), \mathbf{u}_{t}^{h}(t)\right):=\frac{h}{2} \sum_{j=1}^{N} \rho_{j}\left|u_{j}^{\prime}(t)\right|^{2}+\frac{h}{2} \sum_{j=0}^{N} \sigma_{j+1 / 2}\left|\partial_{+}^{h} u_{j}(t)\right|^{2} .
$$


At the same time, the decay property (3.4) is equivalent to the fact that the following observability inequality holds for any solution $\mathbf{u}^{h}(t)$ of the conservative system $(3.6)$ :

$$
\mathcal{E}_{\rho, \sigma}^{h}\left(\mathbf{u}^{h}(0), \mathbf{u}_{t}^{h}(0)\right) \leq C^{\prime}\left(\int_{0}^{T}\left|u_{N+1}^{\prime}(t)\right|^{2} d t+h^{3} \sum_{j=0}^{N-1} \int_{0}^{T}\left|\partial_{+}^{h} u_{j}^{\prime}(t)\right|^{2} d t\right) .
$$

Let us now state the main result of this paper:

Theorem 3.1. $\quad$ a) For all strictly positive coefficients $\rho, \sigma \in C^{2}(0,1)$ and all initial data $\left(\mathbf{u}^{h, 0}, \mathbf{u}^{h, 1}\right) \in$ $\mathbb{R}^{N} \times \mathbb{R}^{N+1}$ in (3.6) such that their total energy $\mathcal{E}_{\rho, \sigma}^{h}\left(\mathbf{u}^{h, 0}, \mathbf{u}^{h, 1}\right)$ is finite, the discrete observability inequality (3.7) holds uniformly in a finite time $T_{\star}^{h}$ that tends to $T_{\star}$ in (2.7) as $h \rightarrow 0$.

b) Equivalently, (3.5) and (3.4) also hold true, uniformly with respect to $h>0$.

Remark 3. Consider $g:[0,1] \rightarrow[0,1]$ to be an increasing $C^{3}(0,1)$ function such that $g^{\prime}(x)>g_{\star}>0$ for all $x \in[0,1]$, a non-uniform grid $\mathcal{G}_{g}^{h}$ of the interval $[0,1]$ constituted by the nodes $g_{j}:=g\left(x_{j}\right)$ and the following viscous numerical approximation of the constant coefficients wave equation $v_{t t}-v_{y y}=0$ on the non-uniform mesh $\mathcal{G}_{g}^{h}$ :

$$
\begin{cases}v_{j}^{\prime \prime}(t)-\frac{\frac{\partial_{+}^{h} v_{j}(t)}{\partial_{+}^{h} g_{j}}-\frac{\partial_{\underline{h}}^{h} v_{j}(t)}{\partial_{-}^{h} g_{j}}}{\partial^{h} g_{j}}=\frac{h^{2} \partial_{2}^{h} v_{j}^{\prime}(t)}{\partial^{h} g_{j}}, & 1 \leq j \leq N, t \in(0, T] \\ v_{0}(t)=\frac{\partial_{+}^{h} v_{N}(t)}{\partial_{+}^{h} g_{N}}+v_{N+1}^{\prime}(t)=0, & t \in[0, T] \\ \mathbf{v}^{h}(0)=\mathbf{v}^{h, 0},\left(\mathbf{v}^{h}\right)^{\prime}(0)=\mathbf{v}^{h, 1} . & \end{cases}
$$

Observe that (3.8) can be also seen as a semi-discretization of the variable coefficients wave equation (1.1) with $\partial^{h} g \sim g^{\prime}=: \rho$ and $1 / \partial_{+}^{h} g \sim 1 / g^{\prime}=: \sigma$ on the uniform mesh $\mathcal{G}^{h}$, so that our result in Theorem 3.1 also applies to system (3.8) and to its conservative version in which the two damping mechanisms represented by the right hand side in the first equation and the second term $v_{N+1}^{\prime}(t)$ in the second equation on the boundary are eliminated.

3.2. Proof of the main result, Theorem 3.1. a) Set $\varphi_{j}:=\varphi\left(x_{j}\right)$, with $\varphi$ as in 2.10). Let us multiply (3.6) by $\varphi_{j} \partial^{h} u_{j}(t)$, add in $1 \leq j \leq N$ and integrate in $t \in(0, T)$. Then

$$
h \sum_{j=1}^{N} \int_{0}^{T} \rho_{j} u_{j}^{\prime \prime}(t) \varphi_{j} \partial^{h} u_{j}(t) d t-h \sum_{j=1}^{N} \int_{0}^{T} \partial_{\sigma}^{h, 2} u_{j}(t) \varphi_{j} \partial^{h} u_{j}(t) d t=0 .
$$

Step 1. Processing of the first term in the left hand side of $(3.9)$. After integration by parts in time, taking real parts and using the identity $2 a b=|a|^{2}+|b|^{2}-|a-b|^{2}$ for all $a, b \in \mathbb{R}$, we obtain

$$
h \sum_{j=1}^{N} \int_{0}^{T} \rho_{j} u_{j}^{\prime \prime}(t) \varphi_{j} \partial^{h} u_{j}(t) d t=\left.\mathcal{X}_{\varphi \rho}^{h}(t)\right|_{0} ^{T}-\frac{1}{4} \sum_{j=1}^{N} \int_{0}^{T}(\varphi \rho)_{j}\left(w_{j+1}(t)-w_{j}(t)\right) d t,
$$

where $w_{j}(t):=\left|u_{j}^{\prime}(t)\right|^{2}+\left|u_{j-1}^{\prime}(t)\right|^{2}-\left|u_{j}^{\prime}(t)-u_{j-1}^{\prime}(t)\right|^{2}$ and

$$
\mathcal{X}_{\alpha}^{h}(t):=h \sum_{j=1}^{N} \alpha_{j} u_{j}^{\prime}(t) \partial^{h} u_{j}(t)
$$

In what follows, we will apply several times the Abbel summation by parts formula:

$$
\sum_{j=\alpha}^{\beta}\left(a_{j+1}-a_{j}\right) b_{j}=a_{\beta+1} b_{\beta+1}-a_{\alpha} b_{\alpha-1}-\sum_{j=\alpha-1}^{\beta} a_{j+1}\left(b_{j+1}-b_{j}\right) \quad \forall \alpha \leq \beta \in \mathbb{Z} .
$$

We first apply formula (3.11) for the last term in the right hand side of 3.10 in the particular case of $\alpha=1, \beta=N, a_{j}=w_{j}(t)$ and $b_{j}=(\varphi \rho)_{j}$. Taking into account the boundary conditions in (3.6), we get 
$w_{N+1}=2\left|u_{N+1}^{\prime}\right|^{2}\left(\right.$ since $\left.u_{N+1}^{\prime}=u_{N}^{\prime}\right)$ and $w_{1}=0$ (since $\left.u_{0}^{\prime}=0\right)$. In this way,

$$
\begin{aligned}
\sum_{j=1}^{N}(\varphi \rho)_{j}\left(w_{j+1}-w_{j}\right) & =2(\varphi \rho)_{N+1}\left|u_{N+1}^{\prime}\right|^{2}-\sum_{j=0}^{N}\left((\varphi \rho)_{j+1}-(\varphi \rho)_{j}\right)\left(\left|u_{j+1}^{\prime}\right|^{2}+\left|u_{j}^{\prime}\right|^{2}\right) \\
& +\sum_{j=0}^{N-1}\left((\varphi \rho)_{j+1}-(\varphi \rho)_{j}\right)\left|u_{j+1}^{\prime}-u_{j}^{\prime}\right|^{2}
\end{aligned}
$$

Due to the boundary condition $u_{0}^{\prime}=0$, the second term in the right hand side of 3.12 verifies the identity

$$
\sum_{j=0}^{N}\left((\varphi \rho)_{j+1}-(\varphi \rho)_{j}\right)\left(\left|u_{j+1}^{\prime}\right|^{2}+\left|u_{j}^{\prime}\right|^{2}\right)=\left((\varphi \rho)_{N+1}-(\varphi \rho)_{N}\right)\left|u_{N+1}^{\prime}\right|^{2}+\sum_{j=1}^{N}\left((\varphi \rho)_{j+1}-(\varphi \rho)_{j-1}\right)\left|u_{j}^{\prime}\right|^{2}
$$

so that we get the following expression for the first term in the left hand side of $(3.9)$ :

$$
\begin{aligned}
& h \sum_{j=1}^{N} \int_{0}^{T} \rho_{j} u_{j}^{\prime \prime}(t) \varphi_{j} \partial^{h} u_{j}(t) d t=\left.\mathcal{X}_{\varphi \rho}^{h}(t)\right|_{0} ^{T}-\frac{(\varphi \rho)_{N+1}+(\varphi \rho)_{N}}{4} \int_{0}^{T}\left|u_{N+1}^{\prime}(t)\right|^{2} d t \\
& +\frac{h}{2} \sum_{j=1}^{N} \int_{0}^{T} \partial^{h}(\varphi \rho)_{j}\left|u_{j}^{\prime}(t)\right|^{2} d t-R_{1}, \text { with } R_{1}:=\frac{h^{3}}{4} \sum_{j=0}^{N-1} \int_{0}^{T} \partial_{+}^{h}(\varphi \rho)_{j}\left|\partial_{+}^{h} u_{j}^{\prime}(t)\right|^{2} d t .
\end{aligned}
$$

Step 2. Processing of the second term in the left hand side of $(3.9)$. First, by simply taking into account that $2 \partial^{h} u_{j}=\partial_{+}^{h} u_{j}+\partial_{+}^{h} u_{j-1}$, we obtain

$$
\begin{aligned}
-h \sum_{j=1}^{N} \int_{0}^{T} \partial_{\sigma}^{h, 2} u_{j}(t) \varphi_{j} \partial^{h} u_{j}(t) d t & =-\frac{1}{2} \sum_{j=1}^{N} \int_{0}^{T} \varphi_{j}\left(\sigma_{j+1 / 2}\left|\partial_{+}^{h} u_{j}(t)\right|^{2}-\sigma_{j-1 / 2}\left|\partial_{+}^{h} u_{j-1}(t)\right|^{2}\right) d t \\
& -\frac{1}{2} \sum_{j=1}^{N} \int_{0}^{T} \varphi_{j}\left(\sigma_{j+1 / 2}-\sigma_{j-1 / 2}\right) \partial_{+}^{h} u_{j}(t) \partial_{-}^{h} u_{j}(t) d t .
\end{aligned}
$$

For the first term in the right hand side of (3.14), we use Abbel formula (3.11) with $\alpha=1, \beta=N$, $a_{j}=\sigma_{j-1 / 2}\left|\partial_{+}^{h} u_{j-1}\right|^{2}$ and $b_{j}=\varphi_{j}$. Taking into account that $a_{N+1}=0$ (since $\left.\partial_{+}^{h} u_{N}=0\right)$, we get

$$
\sum_{j=1}^{N} \varphi_{j}\left(\sigma_{j+1 / 2}\left|\partial_{+}^{h} u_{j}\right|^{2}-\sigma_{j-1 / 2}\left|\partial_{+}^{h} u_{j-1}\right|^{2}\right)=-\varphi_{0} \sigma_{1 / 2}\left|\partial_{+}^{h} u_{0}\right|^{2}-\sum_{j=0}^{N-1} \sigma_{j+1 / 2}\left(\varphi_{j+1}-\varphi_{j}\right)\left|\partial_{+}^{h} u_{j}\right|^{2} .
$$

On the other hand, using the identity $2 a b=|a|^{2}+|b|^{2}-|a-b|^{2}$, holding for all $a, b \in \mathbb{R}$, in the particular case $a=\sigma_{j+1 / 2} \partial_{+}^{h} u_{j}$ and $b=\sigma_{j-1 / 2} \partial_{-}^{h} u_{j}$, we can transform the second term in the right hand side of (3.14) as follows:

$$
\begin{aligned}
\sum_{j=1}^{N} \varphi_{j}\left(\sigma_{j+1 / 2}-\sigma_{j-1 / 2}\right) & \partial_{+}^{h} u_{j} \partial_{-}^{h} u_{j}=\frac{1}{2} \sum_{j=1}^{N} \gamma_{j}\left(\sigma_{j+1 / 2}^{2}\left|\partial_{+}^{h} u_{j}\right|^{2}+\sigma_{j-1 / 2}^{2}\left|\partial_{-}^{h} u_{j}\right|^{2}\right) \\
- & \frac{h^{2}}{2} \sum_{j=1}^{N} \gamma_{j}\left|\partial_{\sigma}^{h, 2} u_{j}\right|^{2} \text {, with } \gamma_{j}:=\varphi_{j} \frac{\sigma_{j+1 / 2}-\sigma_{j-1 / 2}}{\sigma_{j+1 / 2} \sigma_{j-1 / 2}} \quad \forall 1 \leq j \leq N .
\end{aligned}
$$

Since $\partial_{-}^{h} u_{j}=\partial_{+}^{h} u_{j-1}$ and $\partial_{+}^{h} u_{N}=0$, we get

$$
\sum_{j=1}^{N} \gamma_{j}\left(\sigma_{j+1 / 2}^{2}\left|\partial_{+}^{h} u_{j}\right|^{2}+\sigma_{j-1 / 2}^{2}\left|\partial_{-}^{h} u_{j}\right|^{2}\right)=\gamma_{1} \sigma_{1 / 2}^{2}\left|\partial_{+}^{h} u_{0}\right|^{2}+\sum_{j=1}^{N-1}\left(\gamma_{j+1}+\gamma_{j}\right) \sigma_{j+1 / 2}^{2}\left|\partial_{+}^{h} u_{j}\right|^{2} .
$$


Finally, inserting (3.15)-3.17) into 3.14), we obtain

$$
\begin{aligned}
& -h \sum_{j=1}^{N} \int_{0}^{T} \partial_{\sigma}^{h, 2} u_{j}(t) \varphi_{j} \partial^{h} u_{j}(t) d t=\frac{\varphi_{1} \sigma_{1 / 2}^{2}}{4}\left(\frac{1}{\sigma_{1 / 2}}+\frac{1}{\sigma_{3 / 2}}\right) \int_{0}^{T}\left|\partial_{+}^{h} u_{0}(t)\right|^{2} d t \\
& +\frac{h}{2} \sum_{j=1}^{N-1} \int_{0}^{T} \sigma_{j+1 / 2}^{2} \partial_{+}^{h}\left[\frac{\varphi_{j}}{2}\left(\frac{1}{\sigma_{j+1 / 2}}+\frac{1}{\sigma_{j-1 / 2}}\right)\right]\left|\partial_{+}^{h} u_{j}(t)\right|^{2} d t+\frac{h^{2}}{4} \sum_{j=1}^{N} \int_{0}^{T} \gamma_{j}\left|\partial_{\sigma}^{h, 2} u_{j}(t)\right|^{2} d t .
\end{aligned}
$$

Step 3. Equipartition of energy. Note that the last terms in the right hand sides of (3.13) and (3.18) are reminder terms with respect to formulas (A.4) and A.5 (in Appendix A) corresponding to the continuous case. The last term in 3.13 is a discrete version of $-h^{2} \int_{0}^{T} \int_{0}^{1}(\varphi \rho)^{\prime}(x)\left|u_{t x}(x, t)\right|^{2} d x d t / 4$, while the last one in 3.18 is of the form $-h^{2} \int_{0}^{T} \int_{0}^{1} \varphi(x)(1 / \sigma)^{\prime}(x)\left|\left(\sigma u_{x}\right)_{x}(x, t)\right|^{2} d x d t / 4$. However, in the right hand side of the discrete observability inequality (3.7) only the discrete version of the term $h^{2} \int_{0}^{T} \int_{0}^{1}\left|u_{t x}(x, t)\right|^{2} d x d t$ appears, so that firstly we have to express the last terms in (3.13) and (3.18) in terms of the last one in (3.7), modulo eventually some additive reminders. To obtain an equivalent expression of the last term in (3.18), we multiply (3.6) by $\gamma_{j} \partial_{\sigma}^{h, 2} u_{j}(t)$, add in $1 \leq j \leq N$ and integrate in $t \in(0, T)$. After integration by parts in time, we get

$$
\begin{aligned}
& h^{2} \sum_{j=1}^{N} \int_{0}^{T} \gamma_{j}\left|\partial_{\sigma}^{h, 2} u_{j}(t)\right|^{2} d t=h^{2} \sum_{j=1}^{N} \int_{0}^{T} \gamma_{j} \rho_{j} u_{j}^{\prime \prime}(t) \partial_{\sigma}^{h, 2} u_{j}(t) d t \\
& \quad=\left.h^{2} \sum_{j=1}^{N} \gamma_{j} \rho_{j} u_{j}^{\prime}(t) \partial_{\sigma}^{h, 2} u_{j}(t)\right|_{0} ^{T}-h^{2} \sum_{j=1}^{N} \int_{0}^{T} \gamma_{j} \rho_{j} u_{j}^{\prime}(t) \partial_{\sigma}^{h, 2} u_{j}^{\prime}(t) d t .
\end{aligned}
$$

If $a_{j}$ is defined only for values of $j$ between 1 and $N$, then, by applying Abbel formula (3.11) for $\alpha=2$, $\beta=N-1, a_{j}=\sigma_{j-1 / 2} \partial_{+}^{h} g_{j-1}$ and $b_{j}=f_{j}$, we get:

$$
\begin{aligned}
h^{2} \sum_{j=1}^{N} f_{j} \partial_{\sigma}^{h, 2} g_{j} & =h \sum_{j=1}^{N} f_{j}\left(\sigma_{j+1 / 2} \partial_{+}^{h} g_{j}-\sigma_{j-1 / 2} \partial_{+}^{h} g_{j-1}\right) \\
& =h f_{N}\left(\sigma_{N+1 / 2} \partial_{+}^{h} g_{N}-\sigma_{N-1 / 2} \partial_{+}^{h} g_{N-1}\right)+h f_{1}\left(\sigma_{3 / 2} \partial_{+}^{h} g_{1}-\sigma_{1 / 2} \partial_{+}^{h} g_{0}\right) \\
& +h \sum_{j=2}^{N-1} f_{j}\left(\sigma_{j+1 / 2} \partial_{+}^{h} g_{j}-\sigma_{j-1 / 2} \partial_{+}^{h} g_{j-1}\right) \\
& =h f_{N}\left(\sigma_{N+1 / 2} \partial_{+}^{h} g_{N}-\sigma_{N-1 / 2} \partial_{+}^{h} g_{N-1}\right)+h f_{1}\left(\sigma_{3 / 2} \partial_{+}^{h} g_{1}-\sigma_{1 / 2} \partial_{+}^{h} g_{0}\right) \\
& +h f_{N} \sigma_{N-1 / 2} \partial_{+}^{h} g_{N-1}-h f_{1} \sigma_{3 / 2} \partial_{+}^{h} g_{1}-h \sum_{j=1}^{N-1}\left(f_{j+1}-f_{j}\right) \sigma_{j+1 / 2} \partial_{+}^{h} g_{j}
\end{aligned}
$$

and, finally,

$$
h^{2} \sum_{j=1}^{N} f_{j} \partial_{\sigma}^{h, 2} g_{j}=h f_{N} \sigma_{N+1 / 2} \partial_{+}^{h} g_{N}-h f_{1} \sigma_{1 / 2} \partial_{+}^{h} g_{0}-h \sum_{j=1}^{N-1}\left(f_{j+1}-f_{j}\right) \sigma_{j+1 / 2} \partial_{+}^{h} g_{j} .
$$

Apply 3.20 for $f_{j}=\gamma_{j} \rho_{j} u_{j}^{\prime}$ and $g_{j}=u_{j}$. Taking into account that $\partial_{+}^{h} u_{N}=0$, we transform the first term in the right hand side of (3.19) as follows:

$$
h^{2} \sum_{j=1}^{N} \gamma_{j} \rho_{j} u_{j}^{\prime} \partial_{\sigma}^{h, 2} u_{j}=-\mathcal{Y}_{\rho, \sigma}^{h},
$$

with

$$
\mathcal{Y}_{\rho, \sigma}^{h}(t):=h \gamma_{1} \rho_{1} u_{1}^{\prime}(t) \sigma_{1 / 2} \partial_{+}^{h} u_{0}(t)+h \sum_{j=1}^{N-1}\left(\gamma_{j+1} \rho_{j+1} u_{j+1}^{\prime}(t)-\gamma_{j} \rho_{j} u_{j}^{\prime}(t)\right) \sigma_{j+1 / 2} \partial_{+}^{h} u_{j}(t)
$$


Also apply 3.20 for $f_{j}=\gamma_{j} \rho_{j} u_{j}^{\prime}$ and $g_{j}=u_{j}^{\prime}$. Taking into account that $\partial_{+}^{h} u_{N}^{\prime}=0$, from the second term in the right hand side of $(3.19)$ we get:

$$
\begin{aligned}
h^{2} \sum_{j=1}^{N} \gamma_{j} \rho_{j} u_{j}^{\prime} \partial_{\sigma}^{h, 2} u_{j}^{\prime} & =-h^{2} \gamma_{1} \rho_{1} \sigma_{1 / 2}\left|\partial_{+}^{h} u_{0}^{\prime}\right|^{2}-h \sum_{j=1}^{N-1}\left(\gamma_{j+1} \rho_{j+1} u_{j+1}^{\prime}-\gamma_{j} \rho_{j} u_{j}^{\prime}\right) \sigma_{j+1 / 2} \partial_{+}^{h} u_{j}^{\prime} \\
& =-h^{2} \gamma_{1} \rho_{1} \sigma_{1 / 2}\left|\partial_{+}^{h} u_{0}^{\prime}\right|^{2}-\frac{h^{2}}{2} \sum_{j=1}^{N-1}\left(\gamma_{j+1} \rho_{j+1}+\gamma_{j} \rho_{j}\right) \sigma_{j+1 / 2}\left|\partial_{+}^{h} u_{j}^{\prime}\right|^{2} \\
& -\frac{1}{2} \sum_{j=1}^{N-1}\left(\gamma_{j+1} \rho_{j+1}-\gamma_{j} \rho_{j}\right) \sigma_{j+1 / 2}\left(\left|u_{j+1}^{\prime}\right|^{2}-\left|u_{j}^{\prime}\right|^{2}\right) .
\end{aligned}
$$

In the last term in the right hand side of (3.22), for the terms of indices from $j=2$ to $j=N-2$, we apply Abbel formula (3.11) for $\alpha=2, \beta=N-2, a_{j}=\left|u_{j}^{\prime}\right|^{2}, b_{j}=\left(\gamma_{j+1} \rho_{j+1}-\gamma_{j} \rho_{j}\right) \sigma_{j+1 / 2}$ and, taking into account that $u_{N}^{\prime}=u_{N+1}^{\prime}$, we obtain

$$
\begin{aligned}
\sum_{j=1}^{N-1}\left((\gamma \rho)_{j+1}-(\gamma \rho)_{j}\right) \sigma_{j+1 / 2} & \left(\left|u_{j+1}^{\prime}\right|^{2}-\left|u_{j}^{\prime}\right|^{2}\right) \\
& =\left((\gamma \rho)_{N}-(\gamma \rho)_{N-1}\right) \sigma_{N-1 / 2}\left|u_{N+1}^{\prime}\right|^{2}-\left((\gamma \rho)_{2}-(\gamma \rho)_{1}\right) \sigma_{3 / 2}\left|u_{1}^{\prime}\right|^{2} \\
& -\sum_{j=2}^{N-1}\left[\left((\gamma \rho)_{j+1}-(\gamma \rho)_{j}\right) \sigma_{j+1 / 2}-\left((\gamma \rho)_{j}-(\gamma \rho)_{j-1}\right) \sigma_{j-1 / 2}\right]\left|u_{j}^{\prime}\right|^{2} .
\end{aligned}
$$

Finally, by inserting $(3.20)-(3.23)$ into $(3.19)$, we get the following equipartition of energy identity:

$$
h^{2} \sum_{j=1}^{N} \int_{0}^{T} \gamma_{j}\left|\partial_{\sigma}^{h, 2} u_{j}(t)\right|^{2} d t=-\left.\mathcal{Y}_{\rho, \sigma}^{h}(t)\right|_{0} ^{T}+R_{2}+R_{3}+R_{4}
$$

where

$$
\begin{gathered}
R_{2}:=h^{2} \gamma_{1} \rho_{1} \sigma_{1 / 2} \int_{0}^{T}\left|\partial_{+}^{h} u_{0}^{\prime}(t)\right|^{2} d t+\frac{h^{2}}{2} \sum_{j=1}^{N-1} \int_{0}^{T}\left(\gamma_{j+1} \rho_{j+1}+\gamma_{j} \rho_{j}\right) \sigma_{j+1 / 2}\left|\partial_{+}^{h} u_{j}^{\prime}(t)\right|^{2} d t, \\
R_{3}:=\frac{h^{2}}{2} \frac{\partial_{+}^{h}(\gamma \rho)_{N-1} \sigma_{N-1 / 2}}{h} \int_{0}^{T}\left|u_{N+1}^{\prime}(t)\right|^{2} d t
\end{gathered}
$$

and

$$
R_{4}:=-\frac{h^{2}}{2} \frac{\partial_{+}^{h}(\gamma \rho)_{1} \sigma_{3 / 2}}{h} \int_{0}^{T}\left|u_{1}^{\prime}(t)\right|^{2} d t-\frac{h^{2}}{2} \sum_{j=2}^{N-1} \int_{0}^{T} \partial_{\sigma}^{h, 2}(\gamma \rho)_{j}\left|u_{j}^{\prime}(t)\right|^{2} d t .
$$

Step 4. The discrete multiplier identity. By putting together $(3.9),(3.13),(3.18)$ and $(3.24)$, we obtain the following multiplier identity:

$$
E_{c}+E_{p}+E_{r}=\frac{(\varphi \rho)_{N+1}+(\varphi \rho)_{N}}{4} \int_{0}^{T}\left|u_{N+1}^{\prime}(t)\right|^{2} d t-\left.\mathcal{X}_{\varphi \rho}^{h}(t)\right|_{0} ^{T}+\left.\frac{1}{4} \mathcal{Y}_{\rho, \sigma}^{h}(t)\right|_{0} ^{T}+R_{1}-\frac{R_{2}}{4}-\frac{R_{3}}{4}-\frac{R_{4}}{4}
$$

where $\mathcal{X}_{\varphi \rho}^{h}$ and $\mathcal{Y}_{\rho, \sigma}^{h}$ are as in 3.10 and 3.21 , the reminder terms $R_{1}$ to $R_{4}$ are defined in $(3.13)$ and 3.24 and the energy terms $E_{c}, E_{p}, E_{r}$ are as follows:

$$
E_{c}:=\frac{h}{2} \sum_{j=1}^{N} \int_{0}^{T} \partial^{h}(\varphi \rho)_{j}\left|u_{j}^{\prime}(t)\right|^{2} d t
$$




$$
E_{p}:=\frac{h}{2} \sum_{j=1}^{N-1} \int_{0}^{T} \sigma_{j+1 / 2}^{2} \partial_{+}^{h}\left[\frac{\varphi_{j}}{2}\left(\frac{1}{\sigma_{j+1 / 2}}+\frac{1}{\sigma_{j-1 / 2}}\right)\right]\left|\partial_{+}^{h} u_{j}(t)\right|^{2} d t+\frac{h}{2} \int_{0}^{T} \sigma_{1 / 2}\left|\partial_{+}^{h} u_{0}(t)\right|^{2} d t
$$

and

$$
E_{r}:=\frac{\varphi_{1} \sigma_{1 / 2}^{2}}{4}\left(\frac{1}{\sigma_{1 / 2}}+\frac{1}{\sigma_{3 / 2}}\right) \int_{0}^{T}\left|\partial_{+}^{h} u_{0}(t)\right|^{2} d t-\frac{h}{2} \int_{0}^{T} \sigma_{1 / 2}\left|\partial_{+}^{h} u_{0}(t)\right|^{2} d t .
$$

Step 5. Estimates on the energy terms $E_{c}, E_{p}$ and $E_{r}$. Due to the structure of $\varphi$ in 2.10 , even if the coefficients $\rho, \sigma$ in the continuous model are very smooth, $\varphi$ cannot have more than two derivatives in $L^{\infty}(0,1)$ since $\varphi^{\prime}=\psi^{\prime} \varphi$ and $\psi^{\prime}$ involves the absolute values of $\rho^{\prime}$ and $\sigma^{\prime}$ (excepting the situation when both $\rho$ and $\sigma$ are monotonic). By Taylor expansions, for some $\widehat{x}_{j+1 / 2} \in\left(x_{j}, x_{j+1}\right), \widehat{x}_{j+3 / 4} \in\left(x_{j+1 / 2}, x_{j+1}\right)$, $\widehat{x}_{j+1 / 4} \in\left(x_{j}, x_{j+1 / 2}\right), \widehat{x}_{j} \in\left(x_{j-1 / 2}, x_{j+1 / 2}\right)$, we obtain

$$
\partial^{h}(\varphi \rho)_{j}=(\varphi \rho)^{\prime}\left(x_{j}\right)+\frac{h}{4}\left((\varphi \rho)^{\prime \prime}\left(\widehat{x}_{j+1 / 2}\right)-(\varphi \rho)^{\prime \prime}\left(\widehat{x}_{j-1 / 2}\right)\right),
$$

if $(\varphi \rho)^{\prime \prime} \in L^{\infty}(0,1)$, and

$$
\partial_{+}^{h}\left[\frac{\varphi_{j}}{2}\left(\frac{1}{\sigma_{j+1 / 2}}+\frac{1}{\sigma_{j-1 / 2}}\right)\right]=\left(\frac{\varphi}{\sigma}\right)^{\prime}\left(x_{j+1 / 2}\right)+r_{j}^{1},
$$

if $\varphi^{\prime \prime}, \sigma^{\prime \prime} \in L^{\infty}(0,1)$, where

$$
\begin{aligned}
r_{j}^{1} & :=\frac{h}{4} \varphi_{j+1 / 2}\left[\left(\frac{1}{\sigma}\right)^{\prime \prime}\left(\widehat{x}_{j+1}\right)-\left(\frac{1}{\sigma}\right)^{\prime \prime}\left(\widehat{x}_{j}\right)\right]+\frac{h}{8}\left(\frac{1}{\sigma}\right)_{j+1 / 2}\left(\varphi^{\prime \prime}\left(\widehat{x}_{j+3 / 4}\right)-\varphi^{\prime \prime}\left(\widehat{x}_{j+1 / 4}\right)\right) \\
& +\frac{h^{2}}{8}\left(\varphi^{\prime}\right)_{j+1 / 2}\left[\left(\frac{1}{\sigma}\right)^{\prime \prime}\left(\widehat{x}_{j+1}\right)+\left(\frac{1}{\sigma}\right)^{\prime \prime}\left(\widehat{x}_{j}\right)\right]+\frac{h^{2}}{16}\left(\frac{1}{\sigma}\right)_{j+1 / 2}^{\prime}\left(\varphi^{\prime \prime}\left(\widehat{x}_{j+3 / 4}\right)+\varphi^{\prime \prime}\left(\widehat{x}_{j+1 / 4}\right)\right) \\
& +\frac{h^{3}}{32}\left[\varphi^{\prime \prime}\left(\widehat{x}_{j+3 / 4}\right)\left(\frac{1}{\sigma}\right)^{\prime \prime}\left(\widehat{x}_{j+1}\right)-\varphi^{\prime \prime}\left(\widehat{x}_{j+1 / 4}\right)\left(\frac{1}{\sigma}\right)^{\prime \prime}\left(\widehat{x}_{j}\right)\right] .
\end{aligned}
$$

At the points $x \in(0,1)$ where $\sigma^{\prime}(x)=0$ or $\rho^{\prime}(x)=0$, the second-order derivative $\varphi^{\prime \prime}(x)$ has jumps since it depends on $\left(\left|\rho^{\prime}\right|\right)^{\prime}(x)$ and $\left(\left|\sigma^{\prime}\right|\right)^{\prime}(x)$. We define then rigorously $\varphi^{\prime \prime}(x)$ as the generalized derivative (or subderivative, cf. 22], pp. 213) of $\varphi^{\prime}$ taking at the jump points $x$ the set value given by the closed convex hull of $\left\{\varphi^{\prime \prime}(x-), \varphi^{\prime \prime}(x+)\right\}$, where $f(x \pm)$ is the value of $f$ to the left/right of the point $x$.

Thus, by taking into account that $\varphi$ satisfies the second and third inequalities in (A.7), we get the following lower bound on $E_{c}$ and $E_{p}$ :

$$
E_{c} \geq \frac{C_{c}^{h} h}{2} \sum_{j=1}^{N} \int_{0}^{T} \rho_{j}\left|u_{j}^{\prime}(t)\right|^{2} d t, \text { with } C_{c}^{h}:=1-\frac{h}{2} \frac{\left\|(\varphi \rho)^{\prime \prime}\right\|_{L^{\infty}}}{\rho_{\star}},
$$

and

$$
E_{p} \geq \frac{C_{p}^{h} h}{2} \sum_{j=0}^{N-1} \int_{0}^{T} \sigma_{j+1 / 2}\left|\partial_{+}^{h} u_{j}(t)\right|^{2} d t, \text { with } C_{p}^{h}:=1-p^{1}(h)
$$

and

$$
p^{1}(h):=\frac{h}{2} \frac{\|\varphi\|_{L^{\infty}}\left\|(1 / \sigma)^{\prime \prime}\right\|_{L^{\infty}}}{\sigma_{\star}}+\frac{h}{4} \frac{\left\|\varphi^{\prime \prime}\right\|_{L^{\infty}}}{\sigma_{\star}^{2}}+O\left(h^{2}\right) .
$$

Set $C_{\star}^{h}:=\min \left\{C_{c}^{h}, C_{p}^{h}\right\}=1-O(h)$. Taking into account (3.26) and (3.27) and the time conservation of the energy of the solutions of (3.6), we get

$$
E_{c}+E_{p} \geq C_{\star}^{h} T \mathcal{E}_{\rho, \sigma}^{h}\left(\mathbf{u}^{h, 0}, \mathbf{u}^{h, 1}\right) .
$$

Moreover, using the first inequality in (A.7), it is easy to check that, for any $h \leq\left(1+\sigma_{\star} / \sigma^{\star}\right) / 2$, we get that $E_{r}$ mimics the positivity of the last term in the left hand side of (A.6), i.e.,

$$
E_{r} \geq 0 \text {. }
$$


Step 6. Estimates on the reminder terms $R_{1}$ and $R_{2}$. Observe that $R_{1}$ and $R_{2}$ are of the same nature. Therefore, we can write their sum as follows:

$$
R_{1}-\frac{R_{2}}{4}=\frac{h^{3}}{4} \sum_{j=0}^{N-1} \int_{0}^{T} c_{j}\left|\partial_{+}^{h} u_{j}^{\prime}(t)\right|^{2} d t
$$

with

$$
c_{0}:=\partial_{+}^{h}(\varphi \rho)_{0}-\frac{\gamma_{1} \rho_{0} \sigma_{1 / 2}}{h}, \quad c_{j}:=\partial_{+}^{h}(\varphi \rho)_{j}-\frac{\left(\gamma_{j+1} \rho_{j+1}+\gamma_{j} \rho_{j}\right) \sigma_{j+1 / 2}}{2 h}, \quad 1 \leq j \leq N-1,
$$

and $\varphi$ as in 2.10. By taking into account the expression of $\gamma_{j}, 1 \leq j \leq N$, in (3.16), we obtain the following equivalent expressions of the coefficients $c_{j}$ :

$$
c_{0}=\frac{\varphi_{1} \rho_{1} \frac{\sigma_{1 / 2}}{\sigma_{3 / 2}}-\varphi_{0} \rho_{0}}{h} \text { and } c_{j}=\frac{\varphi_{j+1} \rho_{j+1}\left(1+\frac{\sigma_{j+1 / 2}}{\sigma_{j+3 / 2}}\right)-\varphi_{j} \rho_{j}\left(1+\frac{\sigma_{j+1 / 2}}{\sigma_{j-1 / 2}}\right)}{2 h}, \quad 1 \leq j \leq N-1 .
$$

By Taylor expansions around $x_{j+1 / 2}$, we obtain the following expressions of the coefficients $c_{j}, 0 \leq j \leq N-1$, in which $\widehat{x}_{j+1 / 4} \in\left(x_{j}, x_{j+1 / 2}\right), \widehat{x}_{j+3 / 4} \in\left(x_{j+1 / 2}, x_{j+1}\right)$ and $\widehat{x}_{j} \in\left(x_{j-1 / 2}, x_{j+1 / 2}\right)$ :

$$
\begin{aligned}
c_{0} & =\left[\sigma\left(\frac{\varphi \rho}{\sigma}\right)^{\prime}\right]_{1 / 2}+\frac{h}{2}(\sigma \varphi \rho)_{1 / 2}\left(\frac{1}{\sigma}\right)^{\prime}\left(\widehat{x}_{1}\right)+\frac{h}{8}\left((\varphi \rho)^{\prime \prime}\left(\widehat{x}_{3 / 4}\right)-(\varphi \rho)^{\prime \prime}\left(\widehat{x}_{1 / 4}\right)\right)+\frac{h}{2}\left[\sigma(\varphi \rho)^{\prime}\left(\frac{1}{\sigma}\right)^{\prime}\right]_{1 / 2} \\
& +\frac{h^{2}}{4}\left[\sigma(\varphi \rho)^{\prime}\right]_{1 / 2}\left(\frac{1}{\sigma}\right)^{\prime \prime}\left(\widehat{x}_{1}\right)+\frac{h^{2}}{8}(\varphi \rho)^{\prime \prime}\left(\widehat{x}_{3 / 4}\right)\left[\sigma\left(\frac{1}{\sigma}\right)^{\prime}\right]_{1 / 2}+\frac{h^{3}}{16} \sigma_{1 / 2}(\varphi \rho)^{\prime \prime}\left(\widehat{x}_{3 / 4}\right)\left(\frac{1}{\sigma}\right)^{\prime \prime}\left(\widehat{x}_{1}\right)
\end{aligned}
$$

and, for $1 \leq j \leq N-1$,

$$
\begin{aligned}
c_{j} & =\left[\sigma\left(\frac{\varphi \rho}{\sigma}\right)^{\prime}\right]_{j+1 / 2}+\frac{h}{4}(\sigma \varphi \rho)_{j+1 / 2}\left[\left(\frac{1}{\sigma}\right)^{\prime \prime}\left(\widehat{x}_{j+1}\right)-\left(\frac{1}{\sigma}\right)^{\prime \prime}\left(\widehat{x}_{j}\right)\right]+\frac{h}{8}\left((\varphi \rho)^{\prime \prime}\left(\widehat{x}_{j+3 / 4}\right)-(\varphi \rho)^{\prime \prime}\left(\widehat{x}_{j+1 / 4}\right)\right) \\
& +\frac{h^{2}}{8}\left[\sigma(\varphi \rho)^{\prime}\right]_{j+1 / 2}\left[\left(\frac{1}{\sigma}\right)^{\prime \prime}\left(\widehat{x}_{j+1}\right)+\left(\frac{1}{\sigma}\right)^{\prime \prime}\left(\widehat{x}_{j}\right)\right]+\frac{h^{2}}{16}\left((\varphi \rho)^{\prime \prime}\left(\widehat{x}_{j+3 / 4}\right)+(\varphi \rho)^{\prime \prime}\left(\widehat{x}_{j+1 / 4}\right)\right)\left[\sigma\left(\frac{1}{\sigma}\right)^{\prime}\right]_{j+1 / 2} \\
& +\frac{h^{3}}{32} \sigma_{j+1 / 2}\left[(\varphi \rho)^{\prime \prime}\left(\widehat{x}_{j+3 / 4}\right)\left(\frac{1}{\sigma}\right)^{\prime \prime}\left(\widehat{x}_{j+1}\right)-(\varphi \rho)^{\prime \prime}\left(\widehat{x}_{j+1 / 4}\right)\left(\frac{1}{\sigma}\right)^{\prime \prime}\left(\widehat{x}_{j}\right)\right] .
\end{aligned}
$$

The following inequality shows that the main term in each coefficient $c_{j}$ is strictly positive for any $\rho, \sigma$ and $\varphi$ as in 2.10). Thus, for $h$ small enough, all $c_{j}$ are strictly positive. We use here the fact that $\rho \geq \rho_{\star}>0$, $\sigma \geq \sigma_{\star}>0$ and $\left|\sigma^{\prime}\right|,\left|\rho^{\prime}\right|-\rho^{\prime} \geq 0$ :

$$
\left(\frac{\varphi \rho}{\sigma}\right)^{\prime}=\frac{\varphi}{\sigma^{2}}\left(\psi^{\prime} \rho \sigma+\rho^{\prime} \sigma-\rho \sigma^{\prime}\right)=\frac{\varphi}{\sigma^{2}}\left[\rho \sigma+\rho\left(\frac{\left|\sigma^{\prime}\right| \sigma}{\sigma_{\star}}-\sigma^{\prime}\right)+\sigma\left(\frac{\left(\left|\rho^{\prime}\right|-\rho^{\prime}\right) \rho}{\rho_{\star}}+\rho^{\prime}\right)\right] \geq \frac{\varphi \rho}{\sigma}>0 .
$$

Consequently, we get the following estimate on $R_{1}-R_{2} / 4$ :

$$
R_{1}-\frac{R_{2}}{4} \leq \frac{C_{1}^{h} h^{3}}{4} \sum_{j=0}^{N-1} \int_{0}^{T}\left|\partial_{+}^{h} u_{j}^{\prime}(t)\right|^{2} d t
$$

with

$$
C_{1}^{h}:=\left\|\sigma\left(\frac{\varphi \rho}{\sigma}\right)^{\prime}\right\|_{L^{\infty}}+O(h) .
$$

Step 7. Estimates on $\mathcal{X}_{\varphi \rho}^{h}(t)$ and $\mathcal{Y}_{\rho, \sigma}^{h}(t)$. Observe first that, since $\partial^{h} u_{j}=\left(\partial_{+}^{h} u_{j}+\partial_{+}^{h} u_{j-1}\right) / 2, \mathcal{X}_{\varphi \rho}^{h}$ in 3.10 can be rewritten as

$$
\mathcal{X}_{\varphi \rho}^{h}(t)=\frac{h}{2}(\varphi \rho)_{1} u_{1}^{\prime}(t) \partial_{+}^{h} u_{0}(t)+\frac{h}{2} \sum_{j=1}^{N-1}\left((\varphi \rho)_{j+1} u_{j+1}^{\prime}(t)+(\varphi \rho)_{j} u_{j}^{\prime}(t)\right) \partial_{+}^{h} u_{j}(t) .
$$


By applying twice the Cauchy-Schwartz inequality, we obtain

$$
\begin{aligned}
\left|\mathcal{X}_{\varphi \rho}^{h}\right| & \leq \frac{h}{2} \sqrt{\frac{\varphi_{1}^{2} \rho_{1}}{\sigma_{1 / 2}}}\left|\sqrt{\rho_{1}} u_{1}^{\prime}\right|\left|\sqrt{\sigma_{1 / 2}} \partial_{+}^{h} u_{0}\right|+h \sum_{j=1}^{N-1} \alpha_{j+1 / 2} \sqrt{\frac{\rho_{j+1}\left|u_{j+1}^{\prime}\right|^{2}+\rho_{j}\left|u_{j}^{\prime}\right|^{2}}{2}}\left|\sqrt{\sigma_{j+1 / 2}} \partial_{+}^{h} u_{j}\right| \\
& \leq \frac{h}{2} \sum_{j=0}^{N-1} \beta_{j+1 / 2} \sigma_{j+1 / 2}\left|\partial_{+}^{h} u_{j}\right|^{2}+\frac{h}{2} \sum_{j=1}^{N} \beta_{j} \rho_{j}\left|u_{j}^{\prime}\right|^{2},
\end{aligned}
$$

where

$$
\alpha_{j+1 / 2}:=\sqrt{\frac{\varphi_{j+1}^{2} \rho_{j+1}+\varphi_{j}^{2} \rho_{j}}{2 \sigma_{j+1 / 2}}}, \quad \beta_{j+1 / 2}:=\alpha_{j+1 / 2} \quad \forall 1 \leq j \leq N-1, \quad \beta_{1 / 2}:=\frac{1}{2} \sqrt{\frac{\varphi_{1}^{2} \rho_{1}}{\sigma_{1 / 2}}}
$$

and

$$
\beta_{1}:=\frac{1}{2}\left(\alpha_{3 / 2}+\sqrt{\frac{\varphi_{1}^{2} \rho_{1}}{\sigma_{1 / 2}}}\right), \quad \beta_{j}:=\frac{\alpha_{j+1 / 2}+\alpha_{j-1 / 2}}{2} \quad \forall 2 \leq j \leq N-1, \quad \beta_{N}:=\frac{1}{2} \alpha_{N-1 / 2} .
$$

We use the following two Taylor expansions of $\alpha_{j+1 / 2}$, the first one around $x_{j+1 / 2}$ to estimate $\beta_{j+1 / 2}$ and the second one around $x_{j}$ to estimate $\beta_{j}$ :

$$
\alpha_{j+1 / 2}^{2}=\left(\frac{\varphi^{2} \rho}{\sigma}\right)_{j+1 / 2}+\frac{h^{2}}{16 \sigma_{j+1 / 2}}\left[\left(\varphi^{2} \rho\right)^{\prime \prime}\left(\widehat{x}_{j+3 / 4}\right)+\left(\varphi^{2} \rho\right)^{\prime \prime}\left(\widehat{x}_{j+1 / 4}\right)\right]
$$

and

$$
\alpha_{j \pm 1 / 2}^{2}=\left(\frac{\varphi^{2} \rho}{\sigma}\right)_{j} \pm \frac{h}{2}\left(\varphi^{2} \rho\right)_{j}\left(\frac{1}{\sigma}\right)^{\prime}\left(\widehat{x}_{j \pm 1 / 4}\right) \pm \frac{h}{2 \sigma_{j}}\left(\varphi^{2} \rho\right)^{\prime}\left(\widehat{x}_{j \pm 1 / 2}\right)+\frac{h^{2}}{4}\left(\varphi^{2} \rho\right)^{\prime}\left(\widehat{x}_{j \pm 1 / 2}\right)\left(\frac{1}{\sigma}\right)^{\prime}\left(\widehat{x}_{j \pm 1 / 4}\right) .
$$

Then taking into account that $\varphi^{2} \rho / \sigma>0$ on $(0,1)$, we obtain the following upper bound for all $\beta_{j}, 1 \leq j \leq N$, and $\beta_{j+1 / 2}, 0 \leq j \leq N-1$ :

$$
\beta_{j / 2} \leq C_{2}^{h}:=\left\|\varphi \sqrt{\frac{\rho}{\sigma}}\right\|_{L^{\infty}}+O(h) \quad \forall 1 \leq j \leq 2 N .
$$

Consequently,

$$
\left|\mathcal{X}_{\varphi \rho}^{h}(t)\right|_{0}^{T} \mid \leq 2 C_{2}^{h} \mathcal{E}_{\rho, \sigma}^{h}\left(\mathbf{u}^{h, 0}, \mathbf{u}^{h, 1}\right) .
$$

Remark that $2 C_{2}^{h} \rightarrow T_{\star}$ as $h \rightarrow 0$, with $T_{\star}$ as in 2.7.

Note that $\mathcal{Y}_{\rho, \sigma}^{h}(t)$ in 3.21 is of the same nature as $\mathcal{X}_{\varphi \rho}^{h}(t)$ we just estimated, so that the same kind of techniques will be applied. Indeed, by applying the Cauchy-Schwartz inequality as before, we obtain

$$
\begin{aligned}
\left|\mathcal{Y}_{\rho, \sigma}^{h}\right| & \leq h^{2} \sqrt{\frac{\gamma_{1}^{2} \rho_{1} \sigma_{1 / 2}}{h^{2}}}\left|\sqrt{\rho_{1}} u_{1}^{\prime}\right|\left|\sqrt{\sigma_{1 / 2}} \partial_{+}^{h} u_{0}\right|+2 h^{2} \sum_{j=1}^{N-1} \widetilde{\alpha}_{j+1 / 2} \sqrt{\frac{\rho_{j+1}\left|u_{j+1}^{\prime}\right|^{2}+\rho_{j}\left|u_{j}^{\prime}\right|^{2}}{2}}\left|\sqrt{\sigma_{j+1 / 2}} \partial_{+}^{h} u_{j}\right| \\
& \leq h^{2} \sum_{j=0}^{N-1} \widetilde{\beta}_{j+1 / 2} \sigma_{j+1 / 2}\left|\partial_{+}^{h} u_{j}\right|^{2}+h^{2} \sum_{j=1}^{N} \widetilde{\beta}_{j} \rho_{j}\left|u_{j}^{\prime}\right|^{2}
\end{aligned}
$$

where

$$
\widetilde{\alpha}_{j+1 / 2}:=\sqrt{\frac{\left(\gamma_{j+1}^{2} \rho_{j+1}+\gamma_{j}^{2} \rho_{j}\right) \sigma_{j+1 / 2}}{2 h^{2}}}, \quad \widetilde{\beta}_{j+1 / 2}:=\widetilde{\alpha}_{j+1 / 2} \quad \forall 1 \leq j \leq N-1, \quad \widetilde{\beta}_{1 / 2}:=\frac{1}{2} \sqrt{\frac{\gamma_{1}^{2} \rho_{1} \sigma_{1 / 2}}{h^{2}}}
$$

and

$$
\widetilde{\beta}_{1}:=\frac{1}{2}\left(\widetilde{\alpha}_{3 / 2}+\sqrt{\frac{\gamma_{1}^{2} \rho_{1} \sigma_{1 / 2}}{h^{2}}}\right), \quad \widetilde{\beta}_{j}:=\frac{\widetilde{\alpha}_{j+1 / 2}+\widetilde{\alpha}_{j-1 / 2}}{2} \quad \forall 2 \leq j \leq N-1, \quad \widetilde{\beta}_{N}:=\frac{1}{2} \widetilde{\alpha}_{N-1 / 2} .
$$


All $\widetilde{\beta}_{j / 2}, 1 \leq j \leq 2 N$, are finite as $h \rightarrow 0$ since $\gamma_{j}$ in 3.16 approximates $\gamma_{j} \sim h \varphi_{j}(1 / \sigma)_{j}^{\prime}=O(h)$. More precisely, by the same kind of Taylor expansions used above for $\alpha_{j+1 / 2}$, we can prove that

$$
\widetilde{\beta}_{j / 2} \leq \widetilde{C}_{2}^{h}:=\left\|\varphi\left(\frac{1}{\sigma}\right)^{\prime} \sqrt{\rho \sigma}\right\|_{L^{\infty}}+\left\{\begin{array}{ll}
O(h), & \text { if }\left|\sigma^{\prime}\right|>0 \quad \forall x \in[0,1] \\
O(\sqrt{h}), & \text { if }\left|\sigma^{\prime}\right|=0 \text { at some } x \in[0,1]
\end{array} \quad \forall 1 \leq j \leq 2 N .\right.
$$

Finally we get

$$
\frac{1}{4}\left|\mathcal{Y}_{\rho, \sigma}^{h}(t)\right|_{0}^{T} \mid \leq h \widetilde{C}_{2}^{h} \mathcal{E}_{\rho, \sigma}^{h}\left(\mathbf{u}^{h, 0}, \mathbf{u}^{h, 1}\right) .
$$

Step 8. Estimate on the reminder term $R_{4}$ and on the energy on the boundary. The following identity is obvious:

$$
\partial_{\sigma}^{h, 2}(\gamma \rho)_{j}=\partial^{h, 2}(\gamma \rho)_{j} \frac{\sigma_{j+1 / 2}+\sigma_{j-1 / 2}}{2}+\partial^{h}(\gamma \rho)_{j} \frac{\sigma_{j+1 / 2}-\sigma_{j-1 / 2}}{h},
$$

so that

$$
\left|\partial_{\sigma}^{h, 2}(\gamma \rho)_{j}\right| \leq \sigma^{\star}\left|\partial^{h, 2}(\gamma \rho)_{j}\right|+\left.|| \sigma^{\prime}\right|_{L^{\infty}}\left|\partial^{h}(\gamma \rho)_{j}\right| \quad \forall 2 \leq j \leq N-1 .
$$

To estimate the terms $\left|\partial^{h, 2}(\gamma \rho)_{j}\right|$ and $\left|\partial^{h}(\gamma \rho)_{j}\right|$, we first note that, for $\gamma_{j}$ as in (3.16), we obtain the following identity:

$$
(\gamma \rho)_{j}=-(\varphi \rho)_{j} \delta_{j}, \text { with } \delta_{j}:=\frac{1}{\sigma_{j+1 / 2}}-\frac{1}{\sigma_{j-1 / 2}}
$$

and then, using the formula for the discrete Laplacian $\partial^{h, 2}$ of the product of the sequences $\varphi \rho$ and $\delta$, we obtain

$$
\partial^{h, 2}(\gamma \rho)_{j}=-\partial^{h, 2}(\varphi \rho)_{j} \frac{\delta_{j+1}+2 \delta_{j}+\delta_{j-1}}{4}-2 \partial^{h}(\varphi \rho)_{j} \partial^{h} \delta_{j}-\partial^{h, 2} \delta_{j} \frac{(\varphi \rho)_{j+1}+2(\varphi \rho)_{j}+(\varphi \rho)_{j-1}}{4} .
$$

Since $\delta_{j}$ is also a finite difference and, by the hypotheses of Theorem $3.1 \sigma \in C^{2}(0,1)$, we obtain

$$
\partial^{h} \delta_{j}=\frac{h}{2}\left[\partial_{2}^{h}\left(\frac{1}{\sigma}\right)_{j+1 / 2}+\partial_{2}^{h}\left(\frac{1}{\sigma}\right)_{j-1 / 2}\right] \text { and } \partial^{h, 2} \delta_{j}=\partial^{h, 2}\left(\frac{1}{\sigma}\right)_{j+1 / 2}-\partial^{h, 2}\left(\frac{1}{\sigma}\right)_{j-1 / 2} .
$$

Thus

$$
\left|\partial^{h, 2}(\gamma \rho)_{j}\right| \leq 2\|\varphi \rho\|_{L^{\infty}}\left\|\left(\frac{1}{\sigma}\right)^{\prime \prime}\right\|_{L^{\infty}}+h\left[2\left\|(\varphi \rho)^{\prime}\right\|_{L^{\infty}}\left\|\left(\frac{1}{\sigma}\right)^{\prime \prime}\right\|_{L^{\infty}}+\left\|(\varphi \rho)^{\prime \prime}\right\|_{L^{\infty}}\left\|\left(\frac{1}{\sigma}\right)^{\prime}\right\|_{L^{\infty}}\right] .
$$

Similarly, to compute the centered derivative for the product of $\varphi \rho$ and $\delta$, we use the identity

$$
\partial^{h}(\gamma \rho)_{j}=-\partial^{h}((\varphi \rho) \delta)_{j}=-\partial^{h}(\varphi \rho)_{j} \frac{\delta_{j+1}+\delta_{j-1}}{2}-\partial^{h} \delta_{j} \frac{(\varphi \rho)_{j+1}+(\varphi \rho)_{j-1}}{2},
$$

so that

$$
\left|\partial^{h}(\gamma \rho)_{j}\right| \leq h\left[\left\|(\varphi \rho)^{\prime}\right\|_{L^{\infty}}\left\|\left(\frac{1}{\sigma}\right)^{\prime}\right\|_{L^{\infty}}+\|\varphi \rho\|_{L^{\infty}}\left\|\left(\frac{1}{\sigma}\right)^{\prime \prime}\right\|_{L^{\infty}}\right] .
$$

After combining (3.40)-(3.42), we get

$$
\frac{\left|\partial_{\sigma}^{h, 2}(\gamma \rho)_{j}\right|}{\rho_{j}} \leq \frac{2 \sigma^{\star}|| \varphi \rho \|_{L^{\infty}}}{\rho_{\star}}\left\|\left(\frac{1}{\sigma}\right)^{\prime \prime}\right\|_{L^{\infty}}+O(h) \quad \forall 2 \leq j \leq N-1 .
$$

In a similar way, we obtain

$$
\frac{\sigma_{3 / 2}\left|\partial_{+}^{h}(\gamma \rho)_{1}\right|}{h \rho_{1}} \leq \frac{\sigma^{\star}}{\rho_{\star}}\left[\|\varphi \rho\|_{L^{\infty}}\left\|\left(\frac{1}{\sigma}\right)^{\prime \prime}\right\|_{L^{\infty}}+\left\|(\varphi \rho)^{\prime}\right\|_{L^{\infty}}\left\|\left(\frac{1}{\sigma}\right)^{\prime}\right\|_{L^{\infty}}\right]+O(h) .
$$

Finally, we obtain the following estimate of $R_{4}$ :

$$
\frac{\left|R_{4}\right|}{4} \leq h C_{3}^{h} T \mathcal{E}_{\rho, \sigma}^{h}\left(\mathbf{u}^{h, 0}, \mathbf{u}^{h, 1}\right), \quad C_{3}^{h}:=\frac{\sigma^{\star}}{4 \rho_{\star}}\left[2\|\varphi \rho\|_{L^{\infty}}\left\|\left(\frac{1}{\sigma}\right)^{\prime \prime}\right\|_{L^{\infty}}+\left\|(\varphi \rho)^{\prime}\right\|_{L^{\infty}}\left\|\left(\frac{1}{\sigma}\right)^{\prime}\right\|_{L^{\infty}}\right]+O(h) .
$$

Set $C_{4}^{h}:=\left\|(\varphi \rho)^{\prime}\right\|_{L^{\infty}} / 4+h \rho_{\star} C_{3}^{h} / 2$. Similarly to 3.44, we get

$$
\frac{(\varphi \rho)_{N+1}+(\varphi \rho)_{N}}{4} \int_{0}^{T}\left|u_{N+1}^{\prime}(t)\right|^{2} d t+\frac{\left|R_{3}\right|}{4} \leq\left(\frac{(\varphi \rho)(1)}{2}+h C_{4}^{h}\right) \int_{0}^{T}\left|u_{N+1}^{\prime}(t)\right|^{2} d t .
$$



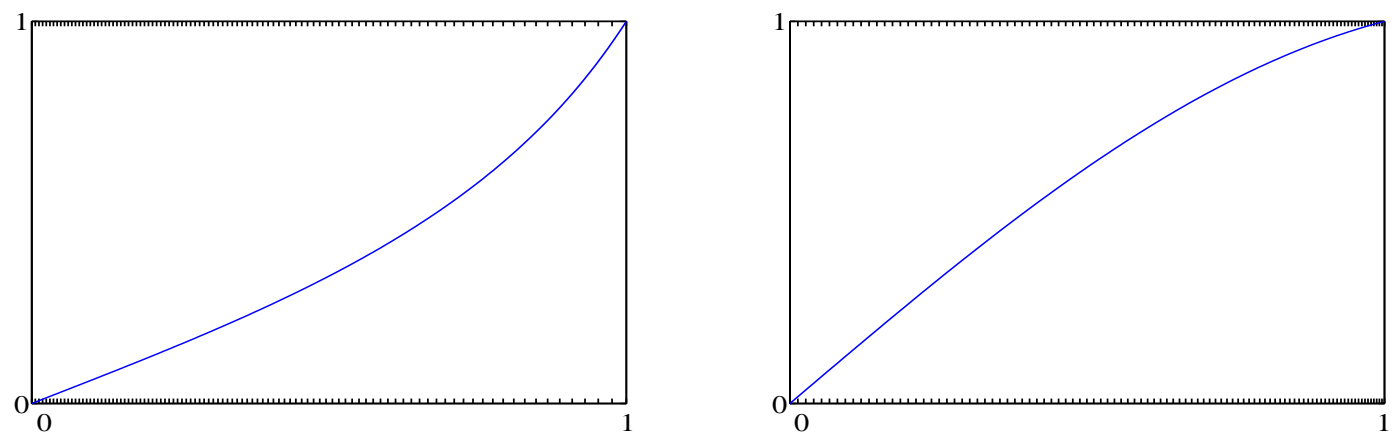

FIgURE 1. The two grids obtained through the applications $g_{1}$ (left) and $g_{2}$ (right).

Replacing the inequalities (3.28), 33.29), 3.33), (3.36), (3.39), (3.45) and (3.46) into the multiplier identity $(3.25)$, we conclude the part a) of Theorem 3.1 by taking the observability time $T>T_{\star}^{h}$ and the observability constant $C^{\prime}$ in (3.7) as follows:

$$
T_{\star}^{h}:=\frac{2 C_{2}^{h}+h \widetilde{C}_{2}^{h}}{C_{\star}^{h}-h C_{3}^{h}} \text { and } C^{\prime}:=\frac{\max \left\{\frac{C_{1}^{h}}{4}, \frac{(\varphi \rho)(1)}{2}+h C_{4}^{h}\right\}}{\left(C_{\star}^{h}-h C_{3}^{h}\right) T-\left(2 C_{2}^{h}+h \widetilde{C}_{2}^{h}\right)} .
$$

3.3. Numerical experiments. As we pointed out in Remark 3, our result in Theorem 3.1 is also valid in the context of numerical discretizations of the constant coefficients wave equation on non-uniform meshes obtained as diffeomorphic transformations of uniform ones through smooth mappings $g$. As we know from [8] or [9, our main result in Theorem 3.1 applied to system (3.8) is also true for the implicit midpoint fully discrete scheme

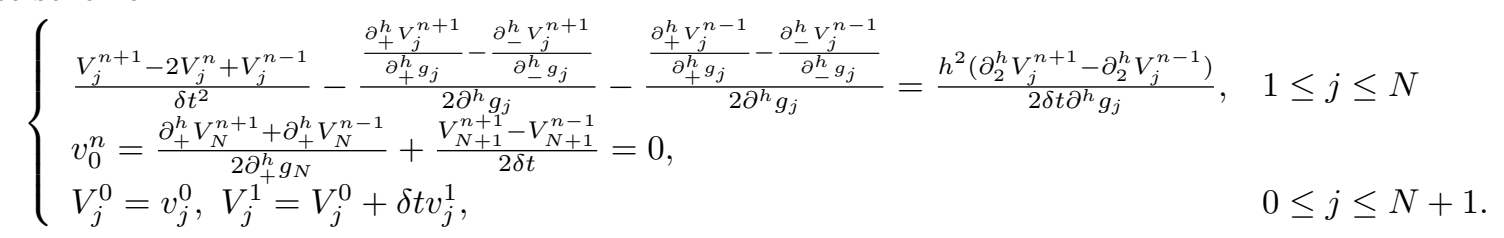

Here, $\delta t$ is the time step, $V_{j}^{n}$ is an approximation of $v_{j}(n \delta t), n \geq 0$, and $\mathbf{v}^{h, i}:=\left(v_{j}^{i}\right)_{0 \leq j \leq N+1}$ are the initial data in 3.8 $\left(i=0,1, v_{0}^{0}=0\right)$. The total energy of the solutions of 3.47),

$$
\begin{aligned}
& \widetilde{\mathcal{E}}_{g}^{h}\left(\mathbf{V}^{h, n}, \mathbf{V}^{h, n-1}\right):= \\
& =\frac{h}{2} \sum_{j=1}^{N} \partial^{h} g_{j}\left|\frac{V_{j}^{n}-V_{j}^{n-1}}{\delta t}\right|^{2}+\frac{h}{4} \sum_{j=0}^{N} \partial_{+}^{h} g_{j}\left(\left|\frac{\partial_{+}^{h} V_{j}^{n}}{\partial_{+}^{h} g_{j}}\right|^{2}+\left|\frac{\partial_{+}^{h} V_{j}^{n-1}}{\partial_{+}^{h} g_{j}}\right|^{2}\right)+\frac{h^{2} \partial_{+}^{h} g_{N}}{2}\left(\left|\frac{\partial_{+}^{h} V_{N}^{n}}{\partial_{+}^{h} g_{N}}\right|^{2}+\left|\frac{\partial_{+}^{h} V_{N}^{n-1}}{\partial_{+}^{h} g_{N}}\right|^{2}\right),
\end{aligned}
$$

satisfies the following dissipation law:

$$
\frac{\widetilde{\mathcal{E}}_{g}^{h}\left(\mathbf{V}^{h, n+1}, \mathbf{V}^{h, n}\right)-\widetilde{\mathcal{E}}_{g}^{h}\left(\mathbf{V}^{h, n}, \mathbf{V}^{h, n-1}\right)}{\delta t}=-\left|\frac{V_{N+1}^{n+1}-V_{N+1}^{n-1}}{2 \delta t}\right|^{2}-h \sum_{j=0}^{N}\left|\frac{V_{j+1}^{n+1}-V_{j+1}^{n-1}}{2 \delta t}-\frac{V_{j}^{n+1}-V_{j}^{n-1}}{2 \delta t}\right|^{2} .
$$

In the numerical simulations, we will consider $v_{j}^{0}=\exp \left(-\gamma\left(x_{j}-g^{-1}\left(y_{0}\right)\right)\right) \cos \left(x_{j} \eta_{0} / h\right), \gamma=h^{-0.9}$, $h=1 / 400, v_{j}^{1}=0$, the final time $T=20$, the time step $\delta t=0.005$ and $\eta_{0} \in\{\pi / 5, \pi / 3, \pi / 2,2 \pi / 3\}$. We will take two non-uniform meshes, $g(x)=g_{1}(x):=\tan (\pi x / 4)$ and $g(x)=g_{2}(x):=2 \sin (\pi x / 6)$. The grid given by $g_{1}$ is finer close to $x=0$ and coarser to the endpoint $x=1$, where the natural damping is acting, while for the grid $g_{2}$ the situation is opposite (see Fig. 1).

In Figures 2 and 4 on the left column, we consider the numerical scheme without the artificial damping given by the right hand side in (3.47) (in that case, we also have to eliminate the last term in the energy $\widetilde{\mathcal{E}}_{g}^{h}$ ), while on the right column we plot the quotient of the energies at time $t=n \delta t$ and $t=0$ for the numerical scheme with the artificial damping. Note that, when the artificial damping term is not added, 


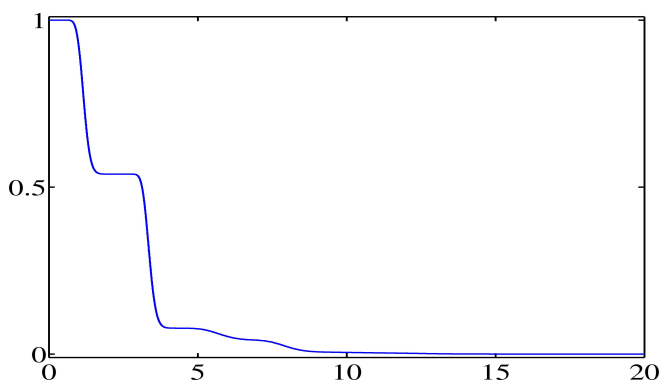

(a) $\eta_{0}=\pi / 5$, no artificial viscosity

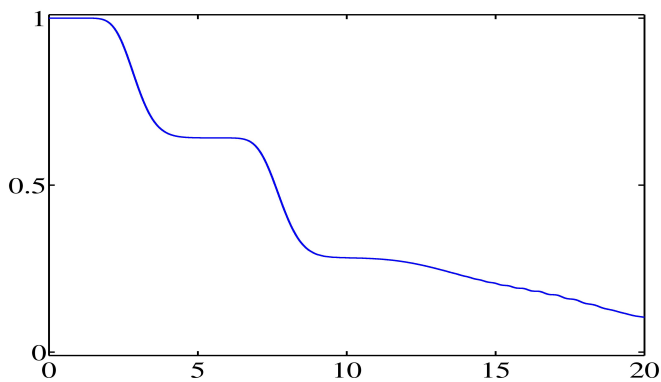

(c) $\eta_{0}=\pi / 3$, no artificial viscosity

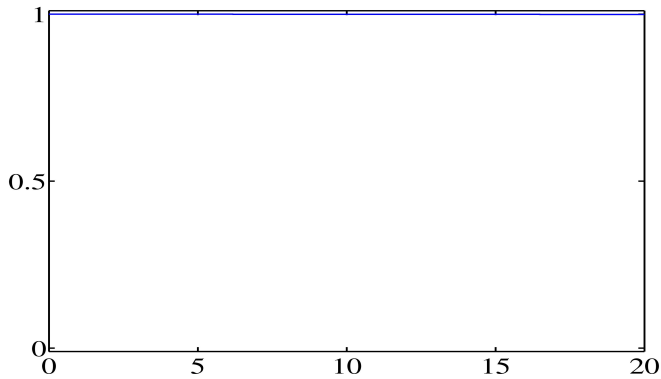

(e) $\eta_{0}=\pi / 2$, no artificial viscosity

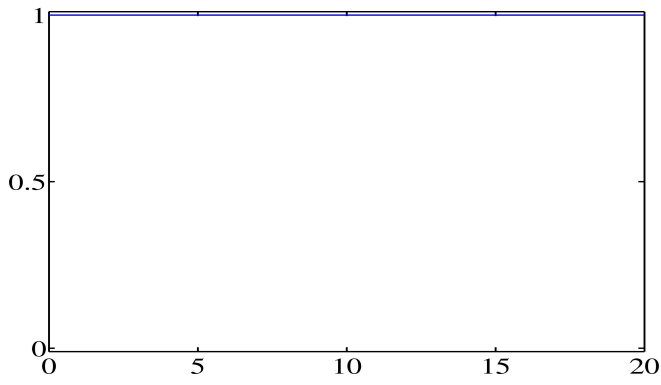

(g) $\eta_{0}=2 \pi / 3$, no artificial viscosity

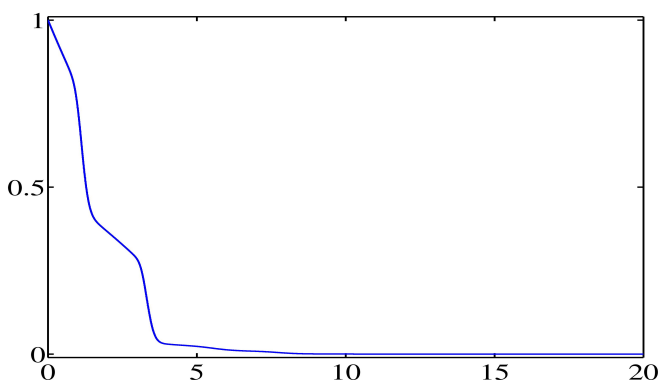

(b) $\eta_{0}=\pi / 5$, with artificial viscosity

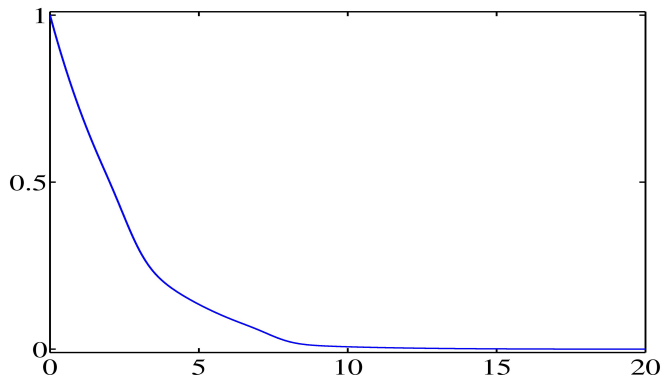

(d) $\eta_{0}=\pi / 3$, with artificial viscosity

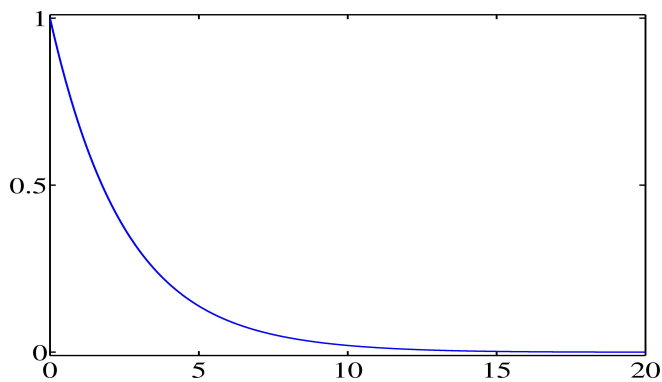

(f) $\eta_{0}=\pi / 2$, with artificial viscosity

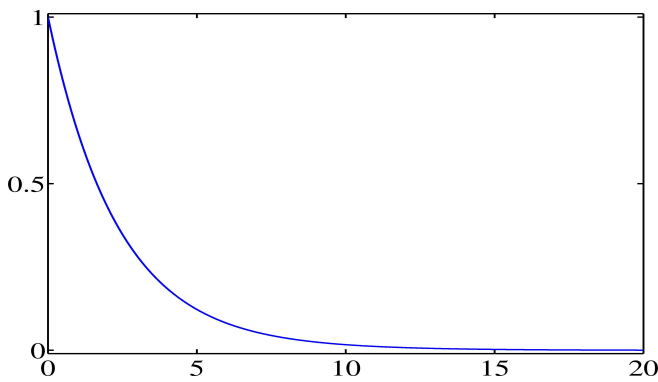

(h) $\eta_{0}=2 \pi / 3$, with artificial viscosity

FIgURE 2. The quotient between the energy at time $n \delta t, \widetilde{\mathcal{E}}_{g}^{h}\left(\mathbf{V}^{h, n}, \mathbf{V}^{h, n-1}\right)$, and the energy at the initial time, $\widetilde{\mathcal{E}}_{g}^{h}\left(\mathbf{V}^{h, 1}, \mathbf{V}^{h, 0}\right)$, for the tangential mesh $g_{1}(x)=\tan (\pi x / 4)$. Each row corresponds to a high frequency oscillation $\eta_{0} \in\{\pi / 5, \pi / 3, \pi / 2,2 \pi / 3\}$ and the left/right column to the numerical approximation without/with numerical viscosity. 


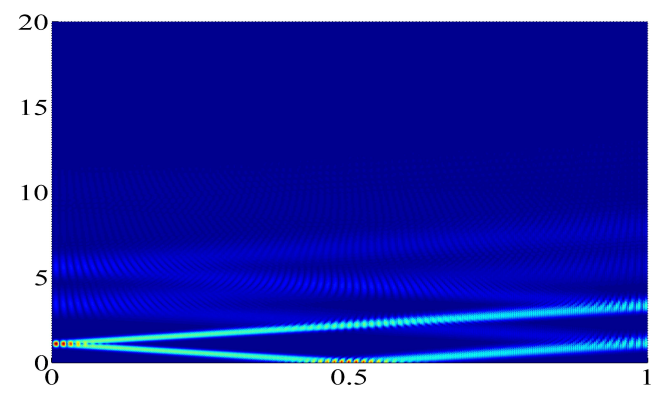

(a) $\eta_{0}=\pi / 5$, no artificial viscosity

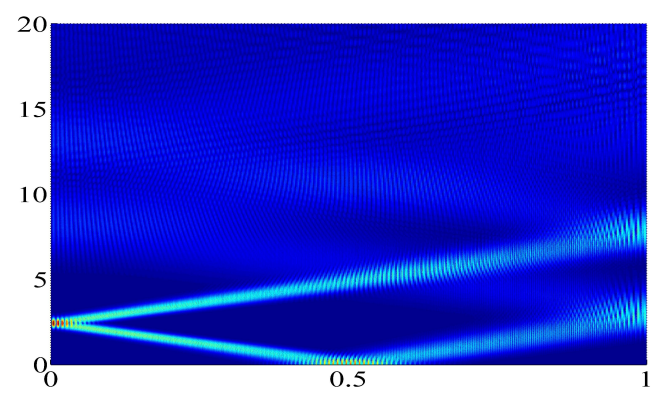

(c) $\eta_{0}=\pi / 3$, no artificial viscosity

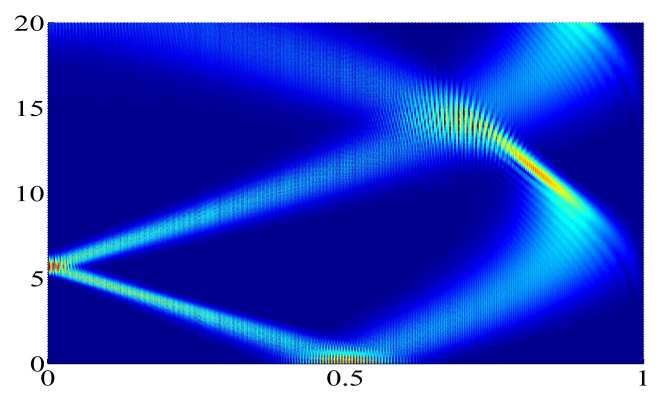

(e) $\eta_{0}=\pi / 2$, no artificial viscosity

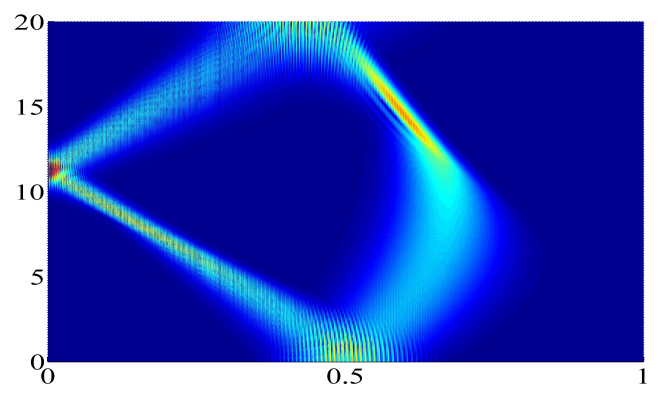

(g) $\eta_{0}=2 \pi / 3$, no artificial viscosity

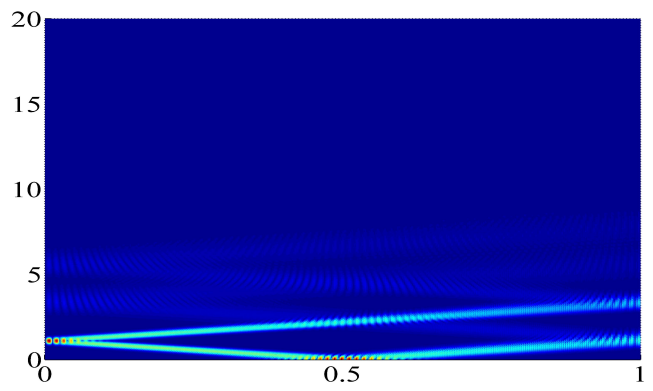

(b) $\eta_{0}=\pi / 5$, with artificial viscosity

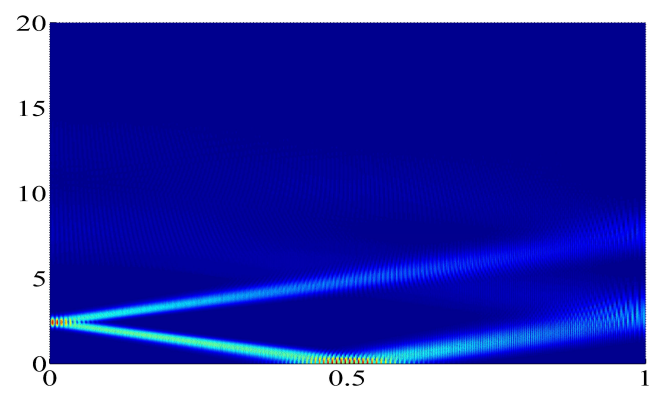

(d) $\eta_{0}=\pi / 3$, with artificial viscosity

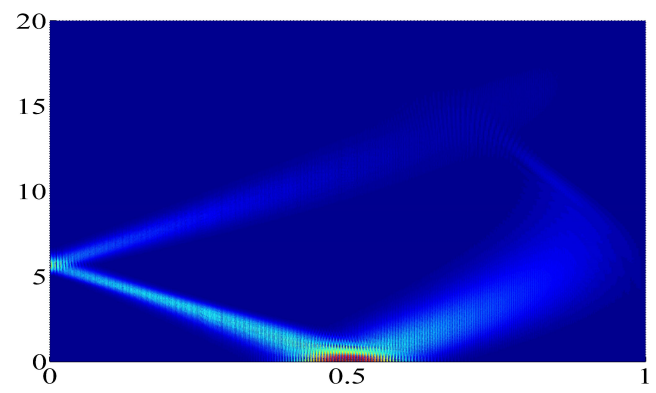

(f) $\eta_{0}=\pi / 2$, with artificial viscosity

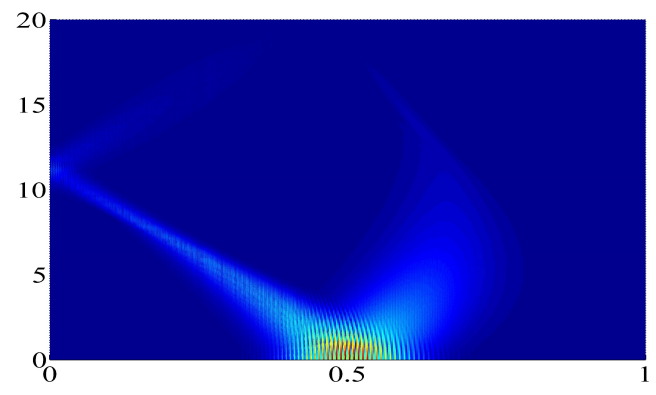

(h) $\eta_{0}=2 \pi / 3$, with artificial viscosity

Figure 3. The solutions of (3.47) on the tangential mesh $g_{1}(x)=\tan (\pi x / 4)$. Each row corresponds to a high frequency oscillation $\eta_{0} \in\{\pi / 5, \pi / 3, \pi / 2,2 \pi / 3\}$ and the left/right column to the numerical approximation without/with numerical viscosity. 


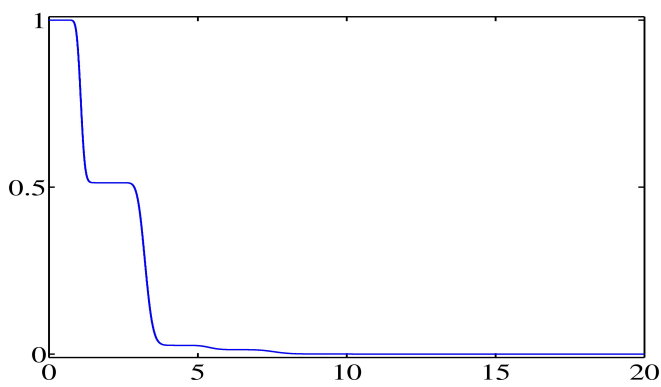

(a) $\eta_{0}=\pi / 5$, no artificial viscosity

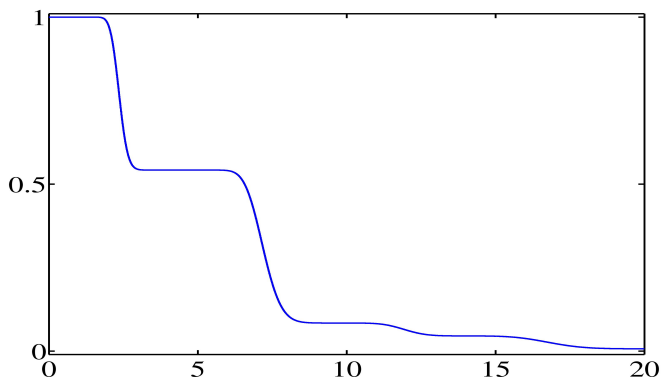

(c) $\eta_{0}=\pi / 3$, no artificial viscosity

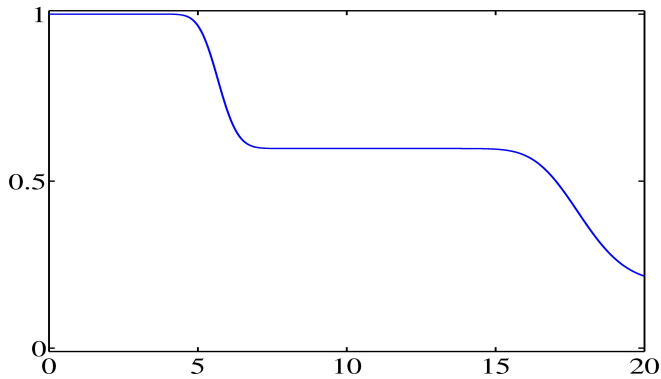

(e) $\eta_{0}=\pi / 2$, no artificial viscosity

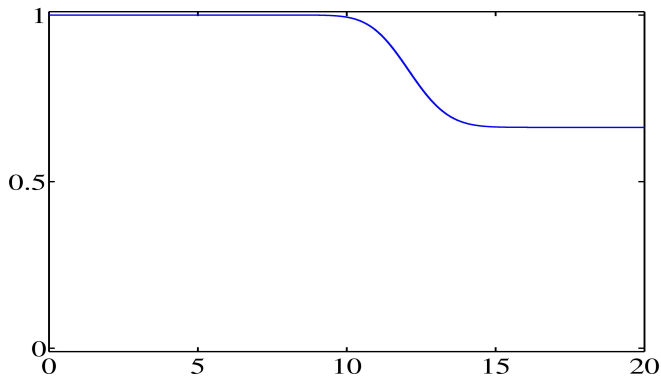

(g) $\eta_{0}=2 \pi / 3$, no artificial viscosity

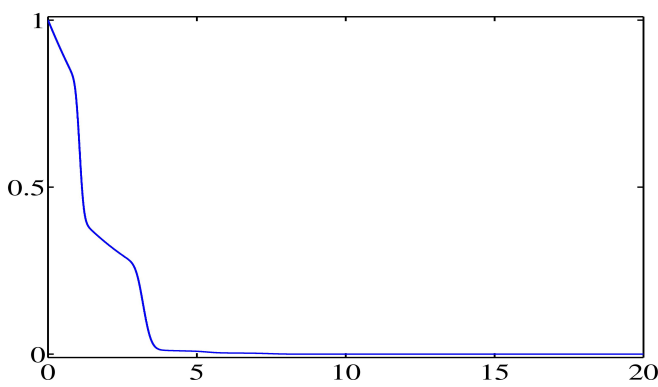

(b) $\eta_{0}=\pi / 5$, with artificial viscosity

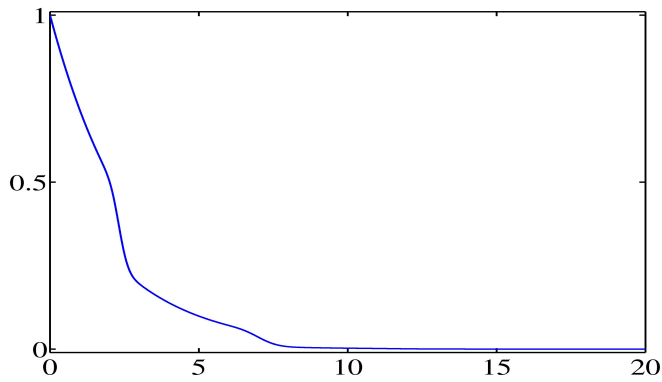

(d) $\eta_{0}=\pi / 3$, with artificial viscosity

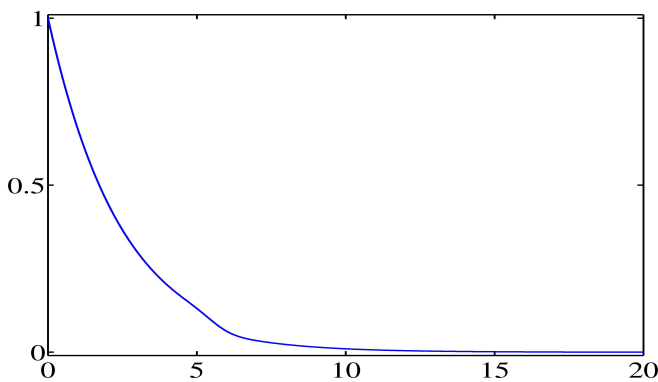

(f) $\eta_{0}=\pi / 2$, with artificial viscosity

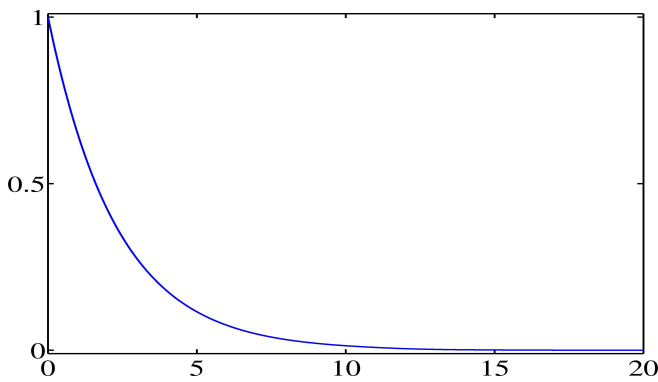

(h) $\eta_{0}=2 \pi / 3$, with artificial viscosity

FIgURE 4. The quotient between the energy at time $n \delta t, \widetilde{\mathcal{E}}_{g}^{h}\left(\mathbf{V}^{h, n}, \mathbf{V}^{h, n-1}\right)$, and the energy at the initial time, $\widetilde{\mathcal{E}}_{g}^{h}\left(\mathbf{V}^{h, 1}, \mathbf{V}^{h, 0}\right)$, for the sinusoidal mesh $g_{2}(x)=2 \sin (\pi x / 6)$. Each row corresponds to a high frequency oscillation $\eta_{0} \in\{\pi / 5, \pi / 3, \pi / 2,2 \pi / 3\}$ and the left/right column to the numerical approximation without/with numerical viscosity. 


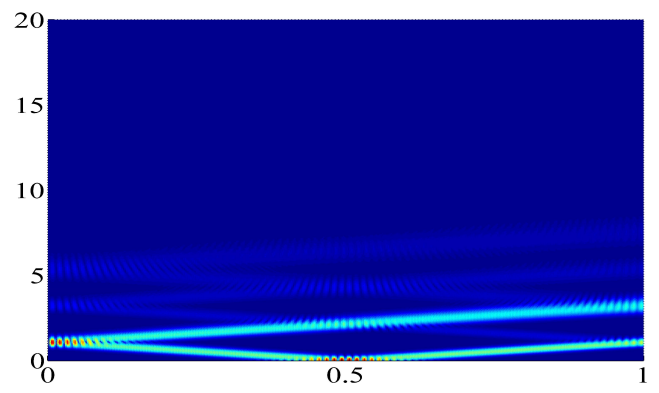

(a) $\eta_{0}=\pi / 5$, no artificial viscosity

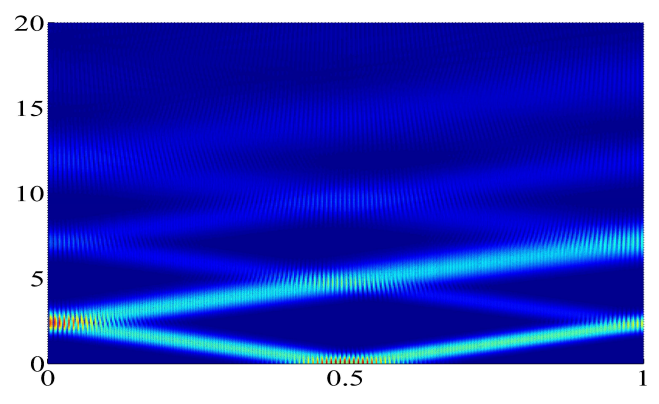

(c) $\eta_{0}=\pi / 3$, no artificial viscosity

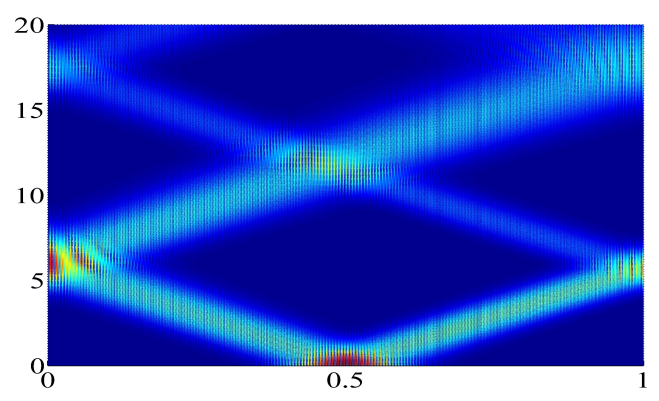

(e) $\eta_{0}=\pi / 2$, no artificial viscosity

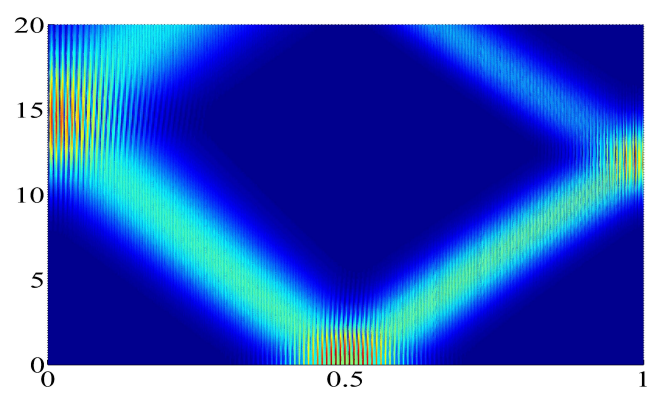

(g) $\eta_{0}=2 \pi / 3$, no artificial viscosity

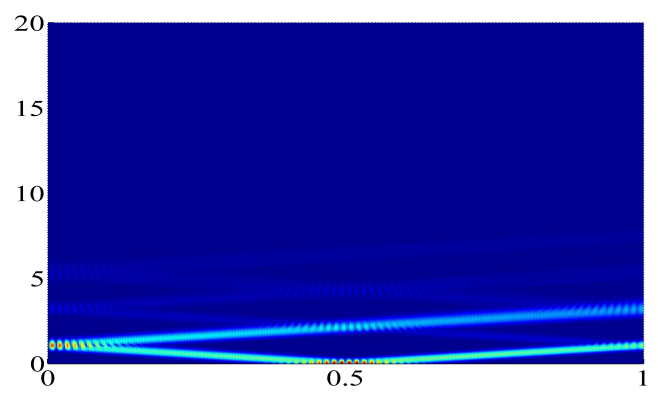

(b) $\eta_{0}=\pi / 5$, with artificial viscosity

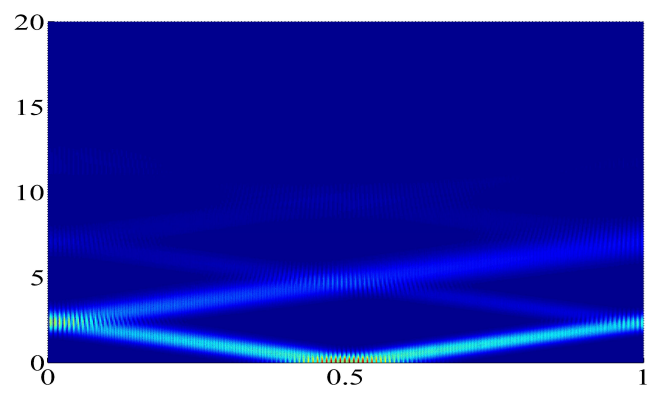

(d) $\eta_{0}=\pi / 3$, with artificial viscosity

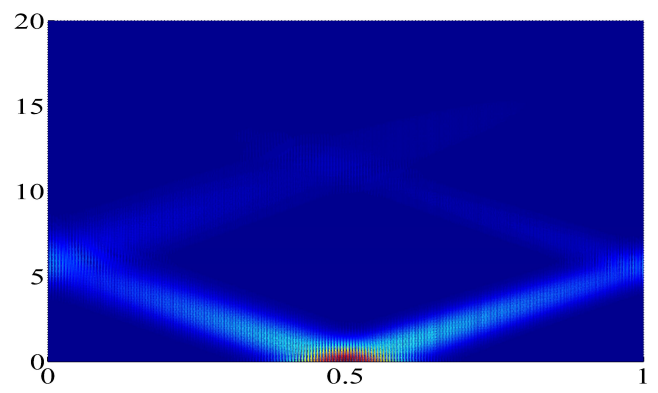

(f) $\eta_{0}=\pi / 2$, with artificial viscosity

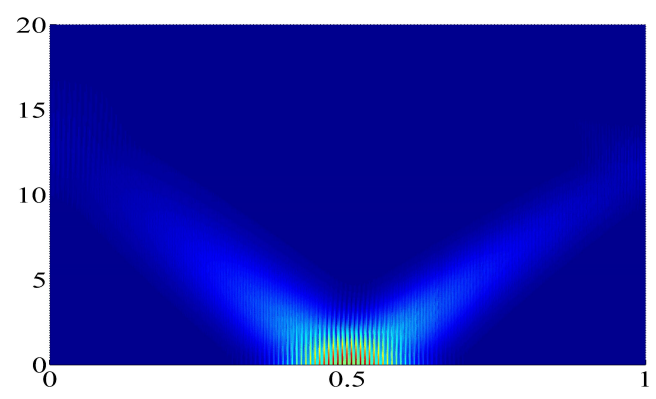

(h) $\eta_{0}=2 \pi / 3$, with artificial viscosity

Figure 5. The solutions of 3.47$)$ on the sinusoidal mesh $g_{2}(x)=2 \sin (\pi x / 6)$. Each row corresponds to a high frequency oscillation $\eta_{0} \in\{\pi / 5, \pi / 3, \pi / 2,2 \pi / 3\}$ and the left/right column to the numerical approximation without/with numerical viscosity. 
for both grids, the quotient of energies has a stepped structure which is due to the fact that the energy of solutions is essentially conserved along the rays of Geometric Optics, except for those time instances at which the support of the solution interacts with the endpoint $x=1$ of the space interval, where the dissipative boundary condition is imposed.

Also remark that the size of the flat areas increases with the frequency, so that the energy of solutions associated to the highest frequency $\left(\eta_{0}=\pi / 2,2 \pi / 3\right)$ wave packets remains essentially constant until the final time $T=20$. This is due to the fact that, as predicted in [20, the corresponding solutions of (3.47) remain concentrated along rays that propagate with a negligible group velocity and that, accordingly, do not interact with the dissipative boundary. In Fig. 3-e, we observe that the initial wave packet splits into two parts, one going to the left and one to the right, and both of them touch the endpoint $x=1$ once, but in a diffractive manner, so that their behaviour is not influenced by the dissipative boundary. In Fig. 3 3 , no one of the two wave packets reaches the dissipative boundary, in Fig. 5 e,g, the right wave packet touches the endpoint $x=1$ and is dissipated, while the left wave packet reaches the dissipative boundary approximately at the final time in Fig. 5 te and does not reaches the dissipative boundary until the final time in Fig. 5rg. On the right columns of Figures $2 \sqrt{5}$ we observe that the damping mechanism is indeed efficient for both grids. Moreover, in the case of added numerical viscosity, the lowest frequencies $\eta_{0}=\pi / 5, \pi / 3$ are dissipated more than exponentially. This is due to the fact that we considered an approximation of the wave equation (1.1) with $\rho=\sigma \equiv 1$, for which the solutions vanish in finite time.

\section{Comments And OPEn PRoblems}

In this paper, we extend to the numerical approximations of the wave equation with regular $\left(C^{2}\right)$ variable coefficients on smooth non-uniform diffeomorphic meshes the results in 24] concerning the efficiency of the vanishing viscosity method to stabilize the numerical schemes for the constant coefficients wave equation on uniform meshes. The method of proof uses multipliers adapted to the variable coefficients as in [4].

We list some open problems related to the content of this article:

- The stabilization problem for the numerical approximations of the variable coefficients multi-dimensional wave equation. Extending the results of this paper to the multi-dimensional case is a challenging open problem. Some of the difficulties encountered when doing that are related to the fact that sidewise energy estimates and multipliers do not yield satisfactory results in the continuous context and that they are hard to adapt to multi-dimensional numerical grids.

- The efficiency of the bi-grid techniques in the stabilization and controllability of the numerical schemes of the wave equation on non-uniform meshes. Bi-grid algorithm was shown to be useful for observability and control problems (cf. 10 and the references therein). But its analysis has been confuted mainly for uniform grids. On the other hand, its use in stabilization problems is to be developed.

- Construct classes of meshes adapted to the damping or control mechanism, for which there is no need to filter the high frequency spurious modes to obtain uniform controllability/stabilization properties of the numerical schemes.

- Numerics for rough coefficients. As explained above, the $1-d$ wave equation with $B V$ coefficients can be observed from the boundary, while here we obtained uniform observability properties for the numerical approximation of the wave equation with smoother coefficients $\left(C^{2}(0,1)\right)$. It remains to analyze the uniform stabilization/control properties of the numerical approximations for the wave equation with less regular coefficients, between $B V(0,1)$ and $C^{2}(0,1)$.

\section{Appendix A. Some technical proofs}

Proof of Theorem 2.2. a) By the method of sidewise energy estimates as in [11, [14, [25] or [26]. Recall that we work under the assumption that the coefficients and initial data in $(2.3)$ are smooth, i.e., $\rho, \sigma \in C^{1}(0,1)$ and $\left(u^{0}, u^{1}\right) \in \mathcal{V}$. For $\varepsilon[u](x, t):=\left(\rho(x)\left|u_{t}(x, t)\right|^{2}+\sigma(x)\left|u_{x}(x, t)\right|^{2}\right) / 2$ being the energy density 
of the solution $u$ of 2.3 , we define the sideways energy

$$
F(x):=\int_{e_{-}(x)}^{e_{+}(x)} \varepsilon[u](x, t) d t
$$

where $e_{+} \geq e_{-}$are two functions to be determined. By applying Leibniz formula for differentiation under the integral sign [15], for which $\varepsilon[u]$ must belong to $C((0,1) \times(0, T)) \cap C^{1}(0,1)$ and $e^{ \pm} \in C^{1}(0,1)$, we obtain

$$
F^{\prime}(x)=A(x)+A_{+}(x)+A_{-}(x), \text { where } A(x):=\frac{1}{2} \int_{e_{-}(x)}^{e_{+}(x)}\left(\rho^{\prime}(x)\left|u_{t}(x, t)\right|^{2}-\sigma^{\prime}(x)\left|u_{x}(x, t)\right|^{2}\right) d t
$$

and

$$
A_{ \pm}(x)= \pm\left(e_{ \pm}\right)^{\prime}(x) \varepsilon[u]\left(x, e_{ \pm}(x)\right) \pm \rho(x) u_{t}\left(x, e_{ \pm}(x)\right) u_{x}\left(x, e_{ \pm}(x)\right)
$$

By choosing $\left(e_{ \pm}\right)^{\prime} \equiv \pm \sqrt{\rho / \sigma}$, we get $A_{ \pm}=0.5 \sqrt{\rho / \sigma}\left|\sqrt{\rho} u_{t}\left(\cdot, e_{ \pm}\right) \pm \sqrt{\sigma} u_{x}\left(\cdot, e_{ \pm}\right)\right|^{2} \geq 0$. Since $A \geq-m F$, with $m:=\max \left\{\left|\rho^{\prime}\right| / \rho,\left|\sigma^{\prime}\right| / \sigma\right\}$, we get $F^{\prime}(x) \geq-m(x) F(x)$. Thus, $F(x) \exp \left(\int_{0}^{x} m(\widetilde{x}) d \widetilde{x}\right)$ is an increasing function, i.e., $F(x) \leq F(1) \exp \left(\int_{x}^{1} m(\widetilde{x}) d \widetilde{x}\right)$ and, by integration in $x \in(0,1)$, we get

$$
\int_{0}^{1} F(x) d x \leq F(1) \int_{0}^{1} \exp \left(\int_{x}^{1} m(\widetilde{x}) d \widetilde{x}\right) d x \leq F(1) \exp \left(\int_{0}^{1} m(x) d x\right) .
$$

We choose $e_{+}(0)=T-\ell$ and $e_{-}(0)=\ell$, so that $e_{+}(1)=T, e_{-}(1)=0$ and $F(1)=\rho(1) \int_{0}^{T}\left|u_{t}(1, t)\right|^{2} d t / 2$ (since $\left.u_{x}(1, t)=0\right)$. In order to ensure the positivity of $F$, we ask $e_{+}(0) \geq e_{-}(0)$, i.e. $T \geq 2 \ell$ (and then, since $e^{+}$and $e^{-}$are increasing/decreasing, $e^{+}>e^{-}$for all $\left.x \in(0,1]\right)$. However, in view of the time conservation of the energy of $u$, in order to prove the observability inequality (2.4), it is sufficient to ask the existence of a rectangle of the form $(0,1) \times\left(t_{-}, t_{+}\right)$, with $t_{-}<t_{+}$, included in the curved trapezoidal region Trap $:=\left\{(x, t), x \in(0,1), e_{-}(x)<t<e_{+}(x)\right\}$ (see the bold curved trapezoidal region in Fig. 6, left). Then $\int_{0}^{1} F(x) d x \geq\left(t_{+}-t_{-}\right) \mathcal{E}_{\rho, \sigma}\left(u^{0}, u^{1}\right)$. Of course, the optimal choice of this rectangle is given by $t_{ \pm}=e_{ \pm}(0)$, so that $0<t_{+}-t_{-}=T-2 \ell$. Since $\exp \left(\int_{0}^{1} m(x) d x\right) \leq \exp \left(T V(\rho, 0,1) / \rho_{\star}+T V(\sigma, 0,1) / \sigma_{\star}\right)$, 2.4 holds with

$$
C^{\prime}:=\frac{\rho(1) \exp \left(T V(\rho, 0,1) / \rho_{\star}+T V(\sigma, 0,1) / \sigma_{\star}\right)}{2(T-2 \ell)} .
$$

The requirement $\varepsilon[u] \in C^{1}(0,1)$ is ensured by the extra regularity assumption imposed on the initial data $\left(u^{0}, u^{1}\right)$ and on the coefficients. Indeed, when the initial data $\left(u^{0}, u^{1}\right) \in \mathcal{V}$, the second-order energy $\mathcal{E}_{\rho, \sigma}^{2}\left(u(\cdot, t), u_{t}(\cdot, t)\right)$ below is also time conservative and finite

$$
\mathcal{E}_{\rho, \sigma}^{2}\left(u(\cdot, t), u_{t}(\cdot, t)\right):=\mathcal{E}_{\rho, \sigma}\left(w(\cdot, t), w_{t}(\cdot, t)\right)=\frac{1}{2} \int_{0}^{1}\left[\rho(x)\left|\partial_{\rho, \sigma}^{2} u_{t}(x, t)\right|^{2}+\sigma(x)\left|\left(\partial_{\rho, \sigma}^{2} u\right)_{x}(x, t)\right|^{2}\right] d x
$$

In particular, there exist two functions $f^{0}, f^{1} \in L^{\infty}\left(0, T ; L^{2}(0,1)\right)$ such that

$$
\left(\sigma u_{t x}\right)_{x}=f^{1} \text { and }\left(\frac{1}{\rho}\left(\sigma u_{x}\right)_{x}\right)_{x}=f^{0} .
$$

Thus,

$$
\left(\sigma u_{x}\right)_{x}(x, t)=\rho(x) \int_{0}^{x} f^{0}\left(x^{\prime}, t\right) d x^{\prime} \in H^{1}(0,1) \text { and } u_{t, x}(x, t)=\frac{1}{\sigma(x)} \int_{0}^{x} f^{1}\left(x^{\prime}, t\right) d x^{\prime} \in H^{1}(0,1)
$$

and, finally, $\sigma u_{x}, u_{t} \in H^{2}(0,1) \subset C^{1}(0,1)$. Since $\sigma, \rho \in C^{1}(0,1)$, we get $\left|\sigma u_{x}\right|^{2} / \sigma, \rho\left|u_{t}\right|^{2}, \varepsilon[u] \in C^{1}(0,1)$.

The direct inequality $(2.6)$ can be obtained by applying the same method of sideways energy estimates within the same class of regular coefficients $\sigma, \rho \in C^{1}(0,1)$ and initial data $\left(u^{0}, u^{1}\right) \in \mathcal{V}$ in $(2.3)$. We only 

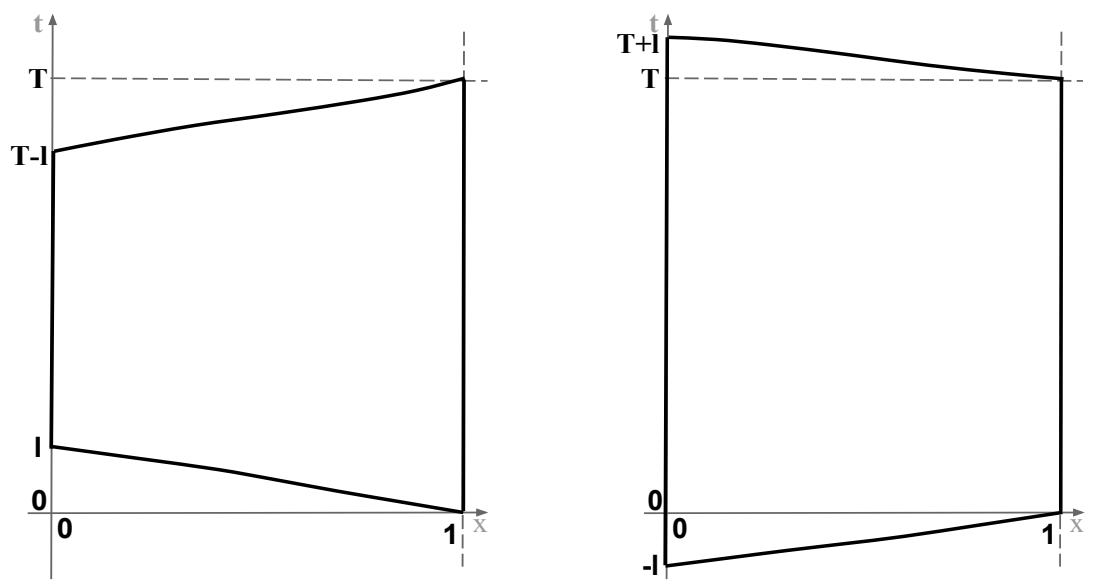

FigurE 6. The two trapezoidal integration domains where the sidewise energy estimates method is applied to prove the observability inequality (left) or the direct inequality (right).

choose $e^{ \pm}$such that $\left(e_{ \pm}\right)^{\prime} \equiv \mp \sqrt{\rho / \sigma}$ and the initial data in $\sqrt{2.3}$ at time $t_{0}=-\ell$. Thus, $A_{ \pm} \leq 0$ and $F^{\prime} \leq m F$, so that, at the end, (2.6) holds with

$$
C^{\prime \prime}:=\frac{2}{\rho(1)} \exp \left(T V(\rho, 0,1) / \rho_{\star}+T V(\sigma, 0,1) / \sigma_{\star}\right)(T+2 \ell) .
$$

By the method of adapted multipliers. Let us consider 2.3 with $\left(u^{0}, u^{1}\right) \in \mathcal{V}$ and strictly positive coefficients $\rho, \sigma \in C^{1}(0,1)$. We multiply 2.3 by $\varphi(x) u_{x}$, with $\varphi$ as in $(2.10$, and integrate in $(0,1) \times(0, T)$ :

$$
0=\int_{0}^{T} \int_{0}^{1} \rho(x) u_{t t}(x, t) \varphi(x) u_{x}(x, t) d x d t-\int_{0}^{T} \int_{0}^{1}\left(\sigma u_{x}\right)_{x}(x, t) \varphi(x) u_{x}(x, t) d x d t
$$

With $\mathcal{X}_{\rho \varphi}(t)$ as in 2.12 and using integrations by parts in both time and space variables in the first term on the right hand side of the above identity, we get:

$$
\begin{aligned}
& \int_{0}^{T} \int_{0}^{1} \rho(x) u_{t t}(x, t) \varphi(x) u_{x}(x, t) d x d t=\left.\mathcal{X}_{\rho \varphi}(t)\right|_{0} ^{T}-\frac{1}{2} \int_{0}^{T} \int_{0}^{1} \rho(x) \varphi\left(\left|u_{t}\right|^{2}\right)_{x}(x, t) d x d t \\
& =\left.\mathcal{X}_{\rho \varphi}(t)\right|_{0} ^{T}-\frac{(\varphi \rho)(1)}{2} \int_{0}^{T}\left|u_{t}(1, t)\right|^{2} d t+\frac{1}{2} \int_{0}^{T} \int_{0}^{1}(\rho \varphi)^{\prime}(x)\left|u_{t}(x, t)\right|^{2} d x d t .
\end{aligned}
$$

From the second term in $\mathrm{A} .3$, we get

$$
\begin{aligned}
& -\int_{0}^{T} \int_{0}^{1}\left(\sigma u_{x}\right)_{x}(x, t) \varphi(x) u_{x}(x, t) d x d t=-\frac{1}{2} \int_{0}^{T} \int_{0}^{1}\left(\frac{\varphi}{\sigma}\right)(x)\left(\left|\sigma u_{x}\right|^{2}\right)_{x}(x, t) d x d t \\
& =\frac{(\varphi \sigma)(0)}{2} \int_{0}^{T}\left|u_{x}(0, t)\right|^{2} d t+\frac{1}{2} \int_{0}^{T} \int_{0}^{1}\left(\frac{\varphi}{\sigma}\right)^{\prime}(x) \sigma^{2}(x)\left|u_{x}(x, t)\right|^{2} d x d t .
\end{aligned}
$$


Putting together the two identities A.4 and A.5 , we obtain

$$
\begin{aligned}
\frac{1}{2} \int_{0}^{T} \int_{0}^{1}\left[(\rho \varphi)^{\prime}(x)\left|u_{t}(x, t)\right|^{2}+\left(\frac{\varphi}{\sigma}\right)^{\prime}(x) \sigma^{2}(x)\left|u_{x}(x, t)\right|^{2}\right] d x d t & +\frac{(\varphi \sigma)(0)}{2} \int_{0}^{T}\left|u_{x}(0, t)\right|^{2} d t \\
& =\frac{(\varphi \rho)(1)}{2} \int_{0}^{T}\left|u_{t}(1, t)\right|^{2} d t-\left.\mathcal{X}_{\rho \varphi}(t)\right|_{0} ^{T} .
\end{aligned}
$$

Let us verify that, for $\varphi$ as in 2.10$)$, the following three inequalities are verified in the sense of $\mathcal{M}(0,1)$, the set of Radon measures on $(0,1)$, which is the dual space of positive $C_{c}^{1}(0,1)$ functions:

$$
\varphi \geq 1, \quad(\varphi \rho)^{\prime} \geq \rho \text { and }\left(\frac{\varphi}{\sigma}\right)^{\prime} \sigma \geq 1 \quad \forall x \in(0,1) .
$$

Since the derivative of the total variation function $T V(a, 0, x)$ is $\left|a^{\prime}(x)\right|$ in a measure sense for any $a \in$ $W^{1,1}(0,1)$, we note that $\psi$ in 2.10 is increasing since $\psi^{\prime}=1+\left|\sigma^{\prime}\right| / \sigma_{\star}+\left(\left|\rho^{\prime}\right|-\rho^{\prime}\right) / \rho_{\star} \geq 1$ and $\psi(0)=0$, so that $\varphi(x) \geq \varphi(0)=1$. On the other hand, since $\rho / \rho_{\star} \geq 1$, then $(\varphi \rho)^{\prime}=\varphi\left(\psi^{\prime} \rho+\rho^{\prime}\right) \geq \varphi\left[\rho+\left(\rho / \rho_{\star}\right)\left(\left|\rho^{\prime}\right|-\rho^{\prime}\right)+\rho^{\prime}\right] \geq$ $\varphi \rho \geq \rho$. Similarly, we get the third inequality in (A.7). Using (A.7) and the time conservation of the total energy for the solution of (2.3), we get the positivity of the second term in the left hand side of (A.6) and the following lower bound on the first term:

$$
\frac{1}{2} \int_{0}^{T} \int_{0}^{1}\left[(\rho \varphi)^{\prime}(x)\left|u_{t}(x, t)\right|^{2}+\left(\frac{\varphi}{\sigma}\right)^{\prime}(x) \sigma^{2}(x)\left|u_{x}(x, t)\right|^{2}\right] d x d t \geq T \mathcal{E}_{\rho, \sigma}\left(u^{0}, u^{1}\right) .
$$

Using the Cauchy-Schwartz inequality, we get

$$
\left|\mathcal{X}_{\varphi \rho}(t)\right| \leq\|\varphi \sqrt{\rho / \sigma}\|_{L^{\infty}} \mathcal{E}_{\rho, \sigma}\left(u^{0}, u^{1}\right)
$$

so that

$$
\left|\mathcal{X}_{\varphi \rho}(t)\right|_{0}^{T}|\leq 2||\varphi \sqrt{\rho / \sigma}|_{L^{\infty}} \mathcal{E}_{\rho, \sigma}\left(u^{0}, u^{1}\right)
$$

Combining identity A.6 with the inequalities A.8 and A.10, we get

$$
\left(T-2 \|\left.\varphi \sqrt{\rho / \sigma}\right|_{L^{\infty}}\right) \mathcal{E}_{\rho, \sigma}\left(u^{0}, u^{1}\right) \leq \frac{(\varphi \rho)(1)}{2} \int_{0}^{T}\left|u_{t}(1, t)\right|^{2} d t,
$$

which concludes the part a) of our result.

c) Let us prove now that (2.4) implies (2.2). We argue by means of a decomposition argument, i.e. we consider 2.3 with the same initial data $\left(v^{0}, v^{1}\right)$ as in 1.1 and the following problem satisfied by the difference $z=v-u$ :

$$
\begin{cases}\rho(x) z_{t t}-\left(\sigma(x) z_{x}\right)_{x}=0, & x \in(0,1), t \in(0, T] \\ z(0, t)=\sigma(1) z_{x}(1, t)+v_{t}(1, t)=0, & t \in[0, T] \\ z(x, 0)=z_{t}(x, 0)=0, & x \in(0,1)\end{cases}
$$

From (2.4) and the fact that $u=v-z$, we get

$$
\mathcal{E}_{\rho, \sigma}\left(v^{0}, v^{1}\right) \leq 2 C^{\prime} \int_{0}^{T}\left|v_{t}(1, t)\right|^{2} d t+2 C^{\prime} \int_{0}^{T}\left|z_{t}(1, t)\right|^{2} d t .
$$

It is enough to prove that

$$
\int_{0}^{T}\left|z_{t}(1, t)\right|^{2} d t \leq C^{\prime \prime} \int_{0}^{T}\left|v_{t}(1, t)\right|^{2} d t
$$


To obtain A.14, we first multiply A.12 by $z_{t}$, integrate in $x \in(0,1)$ and from 0 to $t$, with $t \in(0, T)$, and, taking into account that the initial data in A.12 is the trivial one, we get the identity

$$
\mathcal{E}_{\rho, \sigma}\left(z(\cdot, t), z_{t}(\cdot, t)\right)=-\int_{0}^{t} z_{t}\left(1, t^{\prime}\right) v_{t}\left(1, t^{\prime}\right) d t^{\prime}
$$

The second step is to use sideways energy estimates. More precisely, set $G(x):=\int_{0}^{T} \varepsilon[z](x, t) d t$, where $\varepsilon[z]:=\left(\rho\left|z_{t}\right|^{2}+\sigma\left|z_{x}\right|^{2}\right) / 2$ is the energy density of the solution of A.12. Then, since the initial data in A.12 are the trivial ones, we get

$$
G^{\prime}(x)=\frac{1}{2} \int_{0}^{T}\left(\rho^{\prime}(x)\left|z_{t}(x, t)\right|^{2}-\sigma^{\prime}(x)\left|z_{x}(x, t)\right|^{2}\right) d t+\rho(x) z_{t}(x, T) z_{x}(x, T) .
$$

For $m(x):=\max \left\{\left|\rho^{\prime}(x)\right| / \rho(x),\left|\sigma^{\prime}(x)\right| / \sigma(x)\right\}$, from the previous identity we obtain

$$
G^{\prime}(x) \leq m(x) G(x)+\rho(x)\left|z_{t}(x, T)\right|\left|z_{x}(x, T)\right|
$$

and

$$
G(1) \leq \exp \left(\int_{0}^{1} m(x) d x\right)\left(G(x)+\|\sqrt{\rho / \sigma}\|_{L^{\infty}} \mathcal{E}_{\rho, \sigma}\left(z(\cdot, T), z_{t}(\cdot, T)\right)\right) .
$$

After integrating inequality A.18 in $x \in(0,1)$, we get

$$
G(1) \leq \exp \left(\int_{0}^{1} m(x) d x\right)\left(\int_{0}^{T} \mathcal{E}_{\rho, \sigma}\left(z(\cdot, t), z_{t}(\cdot, t)\right) d t+\|\sqrt{\rho / \sigma}\|_{L^{\infty}} \mathcal{E}_{\rho, \sigma}\left(z(\cdot, T), z_{t}(\cdot, T)\right)\right) .
$$

Note that, by using the boundary condition at $x=1$ in A.12, we obtain

$$
G(1)=\frac{\rho(1)}{2} \int_{0}^{T}\left|z_{t}(1, t)\right|^{2} d t+\frac{1}{2 \sigma(1)} \int_{0}^{T}\left|v_{t}(1, t)\right|^{2} d t .
$$

Using the fact that $\exp \left(\int_{0}^{1} m(x) d x\right) \leq \alpha:=\exp \left(T V(\rho, 0,1) / \rho_{\star}+T V(\sigma, 0,1) / \sigma_{\star}\right)$ and replacing A.15 in A.19, we get

$$
G(1) \leq-\alpha\left[\int_{0}^{T} \int_{0}^{t} z_{t}\left(1, t^{\prime}\right) v_{t}\left(1, t^{\prime}\right) d t^{\prime} d t+\|\sqrt{\rho / \sigma}\|_{L^{\infty}} \int_{0}^{T} z_{t}(1, t) v_{t}(1, t) d t\right] .
$$

By applying Cauchy-Schwartz inequality in $(\mathrm{A} .21$, we get

$$
G(1) \leq \frac{c \epsilon}{2} \int_{0}^{T}\left|z_{t}(1, t)\right|^{2} d t+\frac{c}{2 \epsilon} \int_{0}^{T}\left|v_{t}(1, t)\right|^{2} d t \quad \forall \epsilon>0, \text { with } c:=\alpha\left(T+\|\sqrt{\rho / \sigma}\|_{L^{\infty}}\right) .
$$

Choosing $\epsilon=\rho(1) /(2 c)$ in A.24 and taking into account A.20, we obtain A.14 with

$$
C^{\prime \prime}:=\frac{4}{\rho^{2}(1)}\left(c^{2}-\frac{1}{2} \frac{\rho(1)}{\sigma(1)}\right)>\frac{2\|\sqrt{\rho / \sigma}\|_{L^{\infty}}^{2}}{\rho^{2}(1)}>0 .
$$

To prove that 2.2 implies (2.4), we argue similarly. That is, we consider the same initial data $\left(u^{0}, u^{1}\right)$ in both problems (1.1) and (2.3) and use the same decomposition $v=u+z$ as in the direct implication. It is enough to prove the estimate

$$
\int_{0}^{T}\left|z_{t}(1, t)\right|^{2} d t \leq C^{\prime \prime} \int_{0}^{T}\left|u_{t}(1, t)\right|^{2} d t
$$


Since $v=u+z$, A.21 becomes

$$
\begin{aligned}
& \left(\frac{\rho(1)}{2}+\frac{1}{2 \sigma(1)}+\alpha\|\sqrt{\rho / \sigma}\|_{L^{\infty}}\right) \int_{0}^{T}\left|z_{t}(1, t)\right|^{2} d t+\frac{1}{2 \sigma(1)} \int_{0}^{T}\left|u_{t}(1, t)\right|^{2} d t+\alpha \int_{0}^{T} \int_{0}^{t}\left|z_{t}\left(1, t^{\prime}\right)\right|^{2} d t^{\prime} d t \\
& \leq-\frac{1}{\sigma(1)} \int_{0}^{T} z_{t}(1, t) u_{t}(1, t) d t-\alpha\left(\int_{0}^{T} \int_{0}^{t} z_{t}\left(1, t^{\prime}\right) u_{t}\left(1, t^{\prime}\right) d t^{\prime} d t+\|\sqrt{\rho / \sigma}\|_{L^{\infty}} \int_{0}^{T} z_{t}(1, t) u_{t}(1, t) d t\right) .
\end{aligned}
$$

Then, for all $\epsilon>0$ and $c=1 / \sigma(1)+\alpha\left(T+\|\sqrt{\rho / \sigma}\|_{L^{\infty}}\right)$, we get

$$
\left(\frac{\rho(1)}{2}+\frac{1}{2 \sigma(1)}+\alpha|| \sqrt{\rho / \sigma} \|_{L^{\infty}}\right) \int_{0}^{T}\left|z_{t}(1, t)\right|^{2} d t+\frac{1}{2 \sigma(1)} \int_{0}^{T}\left|u_{t}(1, t)\right|^{2} d t \leq \frac{c \epsilon}{2} \int_{0}^{T}\left|z_{t}(1, t)\right|^{2} d t+\frac{c}{2 \epsilon} \int_{0}^{T}\left|v_{t}(1, t)\right|^{2} d t
$$

By taking $\epsilon=\left(\rho(1) / 2+1 /(2 \sigma(1))+\alpha\|\sqrt{\rho / \sigma}\|_{L^{\infty}}\right) / c$ in the above inequality, we obtain A.23 with

$$
C^{\prime \prime}:=\frac{4}{\left(\rho(1)+1 / \sigma(1)+2 \alpha\|\sqrt{\rho / \sigma}\|_{L^{\infty}}\right)^{2}}\left(c^{2}-\frac{\rho(1)+1 / \sigma(1)+2 \alpha\|\sqrt{\rho / \sigma}\|_{L^{\infty}}}{2 \sigma(1)}\right)>0 .
$$

To prove that 2.2 implies 1.3 , first observe that, due to the dissipation law 1.2 and to 2.2 , we get

$$
\mathcal{E}_{\rho, \sigma}\left(v(\cdot, T), v_{t}(\cdot, T)\right) \leq C \int_{0}^{T}\left|v_{t}(1, t)\right|^{2} d t=C\left(\mathcal{E}_{\rho, \sigma}\left(v^{0}, v^{1}\right)-\mathcal{E}_{\rho, \sigma}\left(v(\cdot, T), v_{t}(\cdot, T)\right)\right),
$$

so that

$$
\mathcal{E}_{\rho, \sigma}\left(v(\cdot, T), v_{t}(\cdot, T)\right) \leq \gamma \mathcal{E}_{\rho, \sigma}\left(v(\cdot, 0), v_{t}(\cdot, 0)\right), \text { with } \gamma:=\frac{C}{C+1} \in(0,1)
$$

and we obtain 1.3 with $M:=1 / \gamma$ and $\omega:=\ln (1 / \gamma) / T$.

To prove that (1.3) implies $(2.2)$, we combine $(1.3)$ with the dissipation law 1.2 and chose $T$ such that $M \exp (-\omega T)<1$.

\section{REFERENCES}

[1] Bardos C., Lebeau G., Rauch J., Sharp sufficient conditions for the observation, control, and stabilization of waves from the boundary, SIAM J. Control Optim., 30(5)(1992), 1024-1065.

[2] Beckermann B., Serra-Capizzano S., On the asymptotic spectrum of finite element matrix sequences, SIAM J. Numer. Anal., 45(2)(2007), 746-769.

[3] Castro C., Zuazua E., Concentration and lack of observability of waves in highly heterogeneous media, Arch. Rat. Mech. Anal., 164(1)(2002), 39-72.

[4] Conrad F., Leblond J., Marmorat J.-P., Boundary control and stabilization of the one-dimensional wave equation, in Boundary control and boundary Variation, Proceedings of IFIP WG 7.2 Conference, Sophia-Antipolis, France, October 15-17, 1990, eds. Zolésio J.P., 142-162.

[5] Cox S., Zuazua E., The rate at which the energy decays in a string damped at one end, Indiana Univ. Math. J., 44(2)(1995), $545-573$.

[6] Ervedoza S., On the mixed finite element method for the $1-d$ wave equation on non-uniform meshes, ESAIM:COCV, 2(2010), 298-326.

[7] Ervedoza S., Zuazua E., On the perfectly matched layers: energy decay for $1-d$ continuous and semi-discrete waves, Numerische Mathematik, 109(4)(2008), 597-634.

[8] Ervedoza S., Zuazua E., Uniformly exponentially stable approximations for a class of damped systems, J. Math. Pures Appl. (9), 91(2009), 20-48.

[9] Ervedoza S., Zuazua E., Uniform exponential decay for viscous damped systems, in Advances in phase space analysis of partial differential equations, 95112, Progress in Nonlinear Differential Equations and their Applications, vol. 78, Birkhäuser Boston, 2009, 95-112.

[10] Ervedoza S., Zuazua E., The wave equation: control and numerics, in Control and stabilization of PDEs, P. M. Cannarsa and J. M. Coron eds., Lecture Notes in Mathematics, Springer Verlag, Vol. 2048, 2012, 245-339.

[11] Fernández-Cara E., Zuazua E., On the null controllability of the one-dimensional heat equation with BV coefficients, Special issue in memory of Jacques-Louis Lions, Comput. Appl. Math., 21(1)(2002), 167-190.

[12] Fomin S. V., Kolmogorov A. N., Introductory real analysis, Dover Publications, New York, 1970. 
[13] Glowinski R., Li C. H., Lions J.-L., A numerical approach to the exact boundary controllability of the wave equation (I). Dirichlet controls: Description of the numerical methods. Japan J. Appl. Math., 7(1990), 1-76.

[14] Haraux A., A generalized internal control for the wave equation in a rectangle, J. Math. Anal. Appl., 153(1)(1990), $190-216$.

[15] Harley F., Differentiation under the integral sign, American Mathematical Monthly, 80(6)(1973), 615-627.

[16] Lagnese J., Control of wave processes with distributed controls supported on a subregion, SIAM J. Cont. Optim., 21(1)(1993), 68-85.

[17] Lions J.L., Contrôlabilité exacte, perturbations et stabilisation des systèmes distribués, vol. 1, Masson, Paris, 1988.

[18] López A., Zuazua E., Uniform null controllability for the one dimensional heat equation with rapidly oscillating periodic density, Annales IHP. Analyse non linéaire, 19(5)(2002), 543-580.

[19] Komornik V., Zuazua E., A direct method for the boundary stabilization of the wave equation, J. Math. Pures Appl. (9), 69(1)(1990), 33-54.

[20] Marica A., Zuazua E., Propagation of $1-d$ waves in regular discrete heterogeneous media: a Wigner measure approach, preprint.

[21] Micu S., Uniform boundary controllability of a semi-discrete $1-d$ wave equation with vanishing viscosity, SIAM J. Cont. Optim., 47(6)(2008), 2857-2885.

[22] Rockafellar R. T., Convex analysis, Princeton Univ. Press, 1970.

[23] Tébou L. R. T., A Carleman estimates based approach for the stabilization of some locally damped semilinear hyperbolic equations, ESAIM: COCV, 14(3)(2008), 561-574.

[24] Tébou L. R. T., Zuazua E., Uniform boundary stabilization of the finite difference space discretization of the $1-d$ wave equation, Adv. Comput. Math., 26(2007), 337-365.

[25] Vancostenoble J., Stabilisation non monotone de systèmes vibrants et contrôlabilité, PhD Thesis, Université de Rennes I, 1998.

[26] Zuazua E., An introduction to the exact controllability for distributed systems, Textos e Notas, 44, C.M.A.F., Universidades de Lisboa, 1990.

[27] Zuazua E., Exponential decay for the semilinear wave equation with locally distributed damping, Comm. Partial Differential Equations, 15(2)(1990), 205-235.

[28] Zuazua E., Exponential decay for the semilinear wave equation with localized damping in unbounded domains, J. Math. Pures Appl. (9), 70(4)(1991), 513-529.

[29] Zuazua E., Propagation, observation, control and numerical approximation of waves, SIAM Review, 47(2)(2005), $197-243$.

Aurora Marica ${ }^{a}$, Enrique Zuazua $^{b, c}$

${ }^{a}$ University of Graz, Institute for Mathematics and Scientific Computing

Heinrichstrasse 36, A-8010, Graz, Austria

${ }^{b}$ BCAM - Basque Center for Applied Mathematics, Alameda Mazarredo 14, 48009, Bilbao, Basque Country, Spain

AND

${ }^{c}$ Ikerbasque - Basque Foundation for Science, Alameda Urquijo 36-5, Plaza Bizkaia, 48011, Bilbao, Basque Country, Spain

E-mail address: aurora.marica@uni-graz.at, zuazua@bcamath.org

$U R L$ : www.bcamath.org/marica/, www.bcamath.org/zuazua/ 